Nouvelles perspectives en sciences sociales

\title{
L'identité corse : une relation récursive entre identités et territoires vécus
}

\section{Marina Casula}

Volume 2, numéro 1, septembre 2006

URI : https://id.erudit.org/iderudit/602454ar

DOI : https://doi.org/10.7202/602454ar

Aller au sommaire du numéro

Éditeur(s)

Prise de parole

ISSN

1712-8307 (imprimé)

1918-7475 (numérique)

Découvrir la revue

Citer cet article

Casula, M. (2006). L'identité corse : une relation récursive entre identités et territoires vécus. Nouvelles perspectives en sciences sociales, 2(1), 9-67.

https://doi.org/10.7202/602454ar d'utilisation que vous pouvez consulter en ligne.

https://apropos.erudit.org/fr/usagers/politique-dutilisation/ 


\title{
L'identité corse : une relation récursive entre identités et territoires vécus
}

\author{
Marina Casula \\ Université de Toulouse 1 \\ CIRESS - LEREPS - GRES, France
}

La «question corse ${ }^{1}$ occupe régulièrement l'espace politique et médiatique français, l'une des problématiques les plus abordées étant celle de la place de la Corse dans la République française avec comme corollaire la définition de l'identité nationale française et le fait pour certains que la reconnaissance du peuple corse, et donc la reconnaissance d'une identité corse existant à côté ou en plus de l'identité nationale française, pourrait remettre en cause l'unité nationale. Les polémiques qui ont vu le jour lors des différentes modifications du statut institutionnel de la Corse (Statut Joxe de 1991, Processus de Matignon en 1999-2002) ${ }^{2}$ nous ont

Voir par exemple et sans exhaustivité Xavier Crettiez, La question corse, Bruxelles, Éditions Complexe, 1999 ; ou encore Wanda Dressler, La Corse en question(s), Ajaccio, Albiana, 2004.

2 Sur le sujet voir, entre autres, Jean-Vitus Albertini et Paul-François Torre, Jospin : le pari corse. Histoire du processus de Matignon, Ajaccio, Albiana Éditions, 2002 ; Jean-Louis Andreani, Pour comprendre la Corse, Paris, Folio-Actuel, Le Monde, 2000 ; Jean-Marie Colombani, Les infortunes de la république, Paris, Grasset, 2000 ; Confluences Méditerranée, «Corse : les enjeux de l'après-Matignon », Paris, L'Harmattan, n 36, Hiver 2000- 
amené à nous poser la question de la définition de l'identité corse. Ce qui suppose répondre à cette interrogation qui semble légitime : « être Corse, c'est quoi ?", ou " comment peut-on être Corse ? " ${ }^{3}$. Notre but n'est pas de définir de façon exhaustive et péremptoire l'identité corse, ni d'en décrire les différents éléments de façon définitive et exhaustive, mais bien de voir comment ils s'articulent. Cette posture nous semble la plus scientifiquement pertinente et la plus éthique, car elle éloigne le spectre d'un certain normativisme identitaire.

Les constatations issues de notre fréquentation du terrain (observation participante et non participante, entretiens) et d'une lecture approfondie des différents ouvrages traitant de l'identité corse, mais aussi de romans (dont on peut dire qu'ils véhiculent et alimentent également les représentations collectives sur la Corse ou d'autres espaces insulaires comme la Sicile, par exemple) nous ont amené à poser et à vérifier l'hypothèse que l'identité corse n'est pas un phénomène univoque mais pluriel.

Nous avons ainsi été amené à constater à de nombreuses reprises que, lorsque nous abordions la question de l'identité corse, les personnes interrogées faisaient très fréquemment référence à leur propre identité personnelle et familiale. Mais, pour autant, cette identité collective affirmée n'est pas la somme ou l'agglomération des identités individuelles des personnes qui s'en revendiquent. L'identité peut être envisagée comme un cadre de référence qui permet aux individus d'orienter leurs choix personnels dans leurs rapports aux autres. $\grave{A}$ un niveau collectif, on assiste au même phénomène où des groupes sociaux, politiques vont élaborer des actions, des projets en fonction des représentations collectives qu'ils partagent quant à leur identité commune. Les développements qui suivent permettront d'approcher ce fonds commun

2001 ; Wanda Dressler, La Corse en question(s), op. cit.; Marianne Lefevre, Géopolitique de la Corse, le modèle républicain en questions, Paris, L'Harmattan, Histoire en perspective, 2000 ; Claude Olivesi, « La Corse contestataire, l'État et l'Europe », dans Fred Reno (dir.), Identité et politique. De la Carä̈be et de l'Europe multiculturelles, Paris, Economica, Caraibe - Amérique Latine, 1995, p. 155-201; Edmond Siméoni, Un combat pour la Corse. Entretiens avec Pierre Dottelonde, Paris, Le Cherche midi, Documents, 2003 ; JeanGuy Talamoni, Ce que nous sommes. Cio che no simu, Préface d'Albert Memmi, Ajaccio, Éditions DCL/Éditions Ramsay, 2001.

3 Antoine-Marie Graziani, "Comment peut-on être Corse? ", Commentaire, Éditions Julliard, vol. $20, \mathrm{n}^{\circ} 80$, hiver 1998, p. 929-939. 
de représentations qui alimentent l'identité collective corse, à partir des entretiens menés auprès d'élus locaux ou de citoyens actifs, militants et autres, mais également à partir d'ouvrages de référence, scientifiques ou romanesques, sur l'identité corse.

Nous verrons plus particulièrement que l'île est au centre des représentations collectives qui organisent et construisent l'identité collective corse. Cette spécificité géographique nourrit donc une conception de l'identité particulière aux îliens en général et aux Corses en particulier (partie 1). Mais par un effet de rétroaction, cette identité alimente à son tour certaines représentations spécifiques sur la façon dont les Corses conçoivent et vivent leur territoire (partie 2).

\section{Insularité et îléité : le territoire nourrit une identité particulière}

Au cours de notre recherche, il est apparu très clairement, et de façon beaucoup plus importante que nous ne l'avions postulé au départ, que l'identité corse repose pour une large partie sur un rapport au territoire. La question de l'insularité a notamment attiré notre attention de façon particulière. Or, parler de l'île en termes conceptuels et pas seulement descriptifs n'est pas chose aisée comme nous le rappelle André-Louis Sanguin. Car « la conscience de l'île présuppose celle du continent »" Cela implique que l'île est le plus souvent pensée dans son rapport au continent et plus rarement étudiée pour elle-même, dans sa singularité. Penser l'île, « faire le tour de l'île », comme souhaite le faire André-Louis Sanguin, implique donc avoir un regard différent.

Les caractéristiques géographiques de la Corse ont eu, et ont toujours, des conséquences sur la façon dont ses habitants occupent l'espace insulaire, se l'approprient. La référence explicite et implicite aux travaux de Braudel est présente dans les représentations collectives des acteurs politiques que nous avons rencontrés, ainsi que de nombreuses allusions à l'œuvre magnifique de Lampedusa : Le Guépard. Ce roman regorge effectivement d'allusions à l'insularité et à la façon dont elle forge les caractères : au fil du récit, le Prince Fabrice Salina évoque auprès de l'un de ses invités ce qu'est pour lui le caractère des Siciliens :

4 André-Louis Sanguin (dir.), " "Faire le tour de l'île"... Quelques jalons pour une géopolitique des insularités", dans Vivre dans une île. Géopolitique des insularités, Paris, L'Harmattan, Géographie et cultures, 1997, p. 11. 
J'ai dit les Siciliens, je devrais ajouter la Sicile, l'atmosphère, le climat, le paysage siciliens. Ce sont ces forces-là qui ont forgé notre âme, au même titre et plus peut-être que les dominations étrangères et les stupres incongrus : ce paysage qui ignore le juste milieu entre la mollesse lascive et la sécheresse infernale ; qui n'est jamais mesquin, banal, prolixe, comme il convient au séjour d'êtres rationnels. [...] Cette violence du paysage, cette cruauté du climat, cette tension perpétuelle de tout ce que l'on voit, ces monuments du passé, magnifiques mais incompréhensibles, parce qu'ils sont construits par d'autres et se dressent autour de nous comme des fantômes grandioses et muets ; tous ces gouvernements débarquant en armes d'on ne sait où, immédiatement servis et détestés, toujours incompris, ne se manifestant que par des œuvres d'art énigmatiques pour nous et par des impôts qui vont grossir ailleurs des caisses étrangères ; tout cela, oui, tout cela a formé notre caractère, qui reste ainsi conditionné par les fatalités extérieures autant que par une terrifiante insularité ${ }^{5}$.

Bien que cet extrait fasse référence à la Sicile de la fin du XIX ${ }^{e}$ siècle, on ne peut qu'être surpris par la force et la résonance qu'il peut avoir, concernant la Corse, son histoire et sa géographie. Les développements suivants seront l'occasion de l'illustrer plus précisément. Cette première partie nous permettra donc de voir ce que signifie et ce qu'implique le fait d'habiter sur une île telle que la Corse et comment les insulaires corses se sont forgés une identité collective à partir des spécificités de leur territoire de vie.

\subsection{L'art d'habiter un espace insulaire ${ }^{6}$}

Réfléchir sur l'espace, c'est accorder une importance à la façon dont un groupe humain ancre ses manifestations, son devenir, son existence dans les relations qu'il entretient avec son milieu naturel qui se présente alors sous la forme d'un espace à parcourir, à habiter, à aménager, à travailler : champs et places publiques, ruelles et chemins, maisons et cabanes, à la fois lieux de pratiques, objets de connaissances, symboles de rapports sociaux, de valeurs communautaires ${ }^{7}$.

5 Guiseppe Tomasi di Lampedusa, Le Guépard, Paris, Seuil, 1959, p. 165-166.

- Allusion au livre de Michel Roux, Inventer un nouvel art d'babiter : le ré-enchantement de l'espace, Paris, L'Harmattan, 2002.

7 Dominique Verdoni, «L'insularité : approche herméneutique de la variation », dans Anne Meisterheim (dir.), Lîlle laboratoire, Colloque de l'Université de Corse, 19-21 juin 1997, Ajaccio, Éditions Alain Piazzola, p. 436. 
Nous verrons ici que, par-delà les contraintes liées à l'insularité, se crée un imaginaire commun, alimenté par les pratiques communes et les sentiments des îliens, qui permettra la construction d'une identité collective. Les exemples pris ici seront principalement tirés de la réalité insulaire corse, mais nous n'excluons pas les références à d'autres espaces insulaires, notamment français ou méditerranéens.

\subsubsection{Ce que « vivre dans une île » veut dire}

L'insularité amène un vécu spécifique. Pour mieux le comprendre, il nous faut prendre connaissance du ressenti des insulaires.

Dans une île, on vit en société. Une société certes très restreinte, très territoriale, très hiérarchisée mais en même temps très solidaire et pleinement ouverte sur le monde extérieure. Ne serait-ce que par nécessité. La plupart des îles où je suis restée [...] ont vu arriver au début des années 1970 des gens qui fuyaient la société de consommation exagérée. Beaucoup sont repartis. Ceux qui sont restés se sont parfaitement intégrés dans le tissu social insulaire. Oh ! Ça ne se fait pas en un jour. [...] Il faut laisser passer une période probatoire de plusieurs mois. Alors on est insulaire. Puis, avec le temps, on devient îlien. Îlois, jamais. Mais quelle importance. On ne choisit pas ses origines ${ }^{8}$.

Or, l'insularité n'est pas qu'une dimension géographique, elle influe également sur l'histoire et l'identité du territoire considéré, comme nous le verrons par la suite. Pour certains acteurs politiques que nous avons rencontrés, la mal-compréhension de cette dimension insulaire et la méconnaissance de ses conséquences sur la vie quotidienne des insulaires expliquent pour une part la difficulté des relations entre la Corse et le continent. Mais faire le constat de cette incompréhension ne suffit pas. En effet, il est plus pertinent de la voir comme un regard particulier que les " continentaux » portent sur l'île et ses habitants.

8 Françoise Sylvestre, «L'âme vagabonde, d'une île à l'autre », Chemins d'étoiles n ${ }^{\circ} 12$ «Îles funestes, îles bienheureuses », août 2004, Paris, Éditions Transboréal, p. 9-10. Journaliste, écrivaine, libraire et éditrice, elle vit aujourd'hui sur l'île-aux-moines, dans le golfe du Morbihan, après avoir vécu de nombreuses années à Belle-île. Sa passion des îles est née de son expérience d'écrivaine : après avoir vécu aux îles Kerguelen pour les besoins de son art, elle a décidé de ne plus vivre ailleurs que sur une île. 
On la regarde vivre comme si, d'ailleurs, elle s'était arrêtée dans le temps et l'espace. L'étonnement, le désarroi ou encore l'agacement des continentaux devant l'immobilisme des îles, devant l'inertie des insulaires et leur difficulté à affronter la "modernité ", traduit bien l'incompréhension d'un rapport au monde si particulier que d'aucuns cherchent à dissimuler sous un discours stéréotypé?

Ce regard a une signification plus profonde quant à la façon de considérer l'île. Les îles sont des mondes en soi, chaque île est un microcosme, ce qui justifie une approche globale de la réalité de chacune $^{10}$. Mais il faut nous interroger sur ce qu'est une île et en quoi l'insularité influe sur la vie des insulaires et conditionne le rapport aux autres.

\subsubsection{Qu'est-ce qu'une île?}

Qu'est-ce qu'une île, véritablement? Un espace isolé du continent sis au milieu d'une étendue d'eau ? Certes! Mais elle est, elles sont bien plus que cela. Car aucune île ne ressemble à une autre : "Les îles se perçoivent comme des centres et non comme des périphéries. Loin de reconnaître leur marginalité par rapport au continent, elles satellisent le monde autour d'elles, s'inscrivant alors en résistance contre ce contexte qui leur semble exercer une pression ${ }^{11}$.

Une difficulté essentielle existe quand on cherche à étudier une ou des îles, c'est l'apparente simplicité de la description que l'on peut en faire, comme le note François Doumenge :

La réalité insulaire, parfaitement définie en apparence par l'interface terre-eau, semble facile à saisir. Cependant on s'aperçoit très vite que

9 Dominique Salini, «L'île entre laboratoire et lab-oratoire ou de la complexité de la mesure », dans Anne Meisterheim (dir.), L'île laboratoire, op. cit., p. 448.

10 Anne Meisterheim, Figures de l'âle, Ajaccio, DCL Éditions, 2001. Sans les développer ici, ces figures sont au nombre de neuf : l'île microcosme, l'île en archipel, l'île solidaire, l'île paradis, l'île labyrinthe, l'île des masques, l'île conservatoire, l'île laboratoire, l'île système. L'auteur a forgé ces figures « pour essayer de saisir toute la complexité des îles, des insulaires et de leurs relations diverses, relations confrontées aux représentations de l'île, confrontées aux discours sur l'insularité et les insulaires » (p. 19).

11 Cité par Jean-Louis Andreani, Pour comprendre la Corse, Folio-Actuel, Le Monde, 1999, p. 19. 


\begin{abstract}
le fait d'être une terre émergée au-dessus du niveau de la plus haute mer, et entourée de toute part, fournit simplement un critère relatif qui doit être apprécié à la fois en fonction de ses caractères spatiaux propres (dimensions, formes, volumes) et par rapport aux autres terres émergées. L'ensemble des équilibres physiques, biologiques, socioéconomiques et géo-politiques qui reposent sur ces bases et ces relations prend le nom d'insularité. Ces caractères d'insularité peuvent contribuer à donner des phénomènes limites d'isolement, de fragmentation, faisant apparaitre des situations d'insularisme ${ }^{12}$.
\end{abstract}

Il faut donc considérer le fait que chaque île doit être approchée de façon spécifique, car il existe une multitude de situations d'insularités qui ne sont pas toujours comparables. Il existe plusieurs typologies des espaces insulaires ${ }^{13}$. Mais elles n'abordent pas vraiment la question des vécus particuliers, des sentiments d'identité partagée par les insulaires. Bien sûr, ce vécu peut être différent selon le sexe ou l'âge des îliens, leur ancienneté dans le lieu, mais, quoi qu'il en soit, tous restent attachés à leur île ${ }^{14}$. Il sera également totalement différent pour les îles du Pacifique ou d'ailleurs qui ont subi les conquêtes des Européens et des flux de migrations qui ont totalement transformé les populations d'origine, quand elles ne les ont pas décimées ${ }^{15}$.

C'est pourquoi la typologie suivante, construite par Anne Meistersheim, nous semble plus intéressante à mobiliser dans le cadre du projet de connaissance du phénomène identitaire corse que nous construisons ici. Il apparaît donc trois notions pour évoquer l'île. L'insularité pour désigner « tout ce qui relève de la géographie et de l'économie, toutes les

12 François Doumenge, «Les îles et les micro-états insulaires », Hérodote : revue de géographie et de géographie politique, $\mathrm{n}^{\mathrm{os}} 37-38,2^{\mathrm{e}}$ et $3^{\mathrm{c}}$ trimestres 1985, cité par Joseph Martinetti, Insularité et marginalité en Méditerranée occidentale : l'exemple de la Corse, Ajaccio, Le Signet, 1991, p. 13.

1.3 Voir par exemple Joseph Martinetti, Insularité et maryinalité..., ibid., p. 24-25 ou encore André-Louis Sanguin, " "Faire le tour de l'île"... », dans André-Louis Sanguin (dir.), Viure dans une ille, op. cit., p. 15.

14 Jean-Pierre Castelain, "Insularités ", Ethnologie Française, vol. XXXIV, n" 1, 2004, p. 99.

15 La question des univers insulaires pacifiques et océaniens est tellement vaste que nous ne la développerons pas davantage ici. Nous renvoyons le lecteur à l'ouvrage de Dominique Guillaud, Christian Huetz De Lemps et Olivier Sevin (dir.), Îles rêvées. Territoires et identités en crise dans le Pacifique insulaire, Paris, Presses de l'Université de Paris-Sorbonne, 2003. 


\section{6 \\ NPSS, VOLUME 2, NUMÉRO 1, 2006}

données que l'on peut aborder à partir de mesures et de chiffres et pour lesquelles on peut construire des "indices". Et on peut parler d'insularité notamment à propos de développement économique ${ }^{16}$. L'insularisme pour appréhender « l'ensemble des phénomènes qui relèvent du domaine politique, qu'il s'agisse du comportement politique des insulaires ou de la situation géopolitique des îles, de leurs institutions particulières, des interactions entre État central et îles, des relations entre îles en situation d'archipel $»^{17}$. L'îlété pour aborder « le vécu des insulaires, leur culture, leur imaginaire, tous les comportements induits par la nature particulière de l'espace insulaire, du temps, de la société, et qui traverse ainsi et soustend tous les phénomènes ${ }^{18}$. Ces notions sont complémentaires et représentent trois aspects de ce que nous pouvons appeler la culture insulaire.
Au-delà des traits caractéristiques de l'aire culturelle à laquelle se rattache chaque île [...], il existe dans toutes les îles, ou plus précisément dans toutes les sociétés insulaires, des traits communs, traits déterminés par la petite taille de l'espace qui, par exemple, impose aux insulaires une multiplicité de rôles, une vie sociale complexe mais aussi une solidarité et un lien social très vivants ${ }^{19}$.

Nous pouvons dès lors voir l'îléité comme une boucle récursive entre une identité et un territoire. La boucle récursive est une « notion essentielle pour concevoir les processus d'auto-organisation et d'autoproduction. Elle constitue un circuit où les effets rétroagissent sur les causes, où les produits sont eux-mêmes producteurs de ce qui les produit. Cette notion dépasse la conception linéaire de la causalité ${ }^{20}$. C'est-à-dire ici que l'identité collective va engendrer une certaine représentation du territoire (en insistant sur certaines caractéristiques de ce territoire) et la représentation du territoire va à son tour engendrer certaines caractéristiques qui vont participer à la construction d'une identité collective.

16 Anne Meistersheim, Figures de l'âle, op. cit., p. 20.

17 Ibid., p. 20-21.

18 Ibid., p. 21.

19 Ibid., p. 21.

20 Edgar Morin, La Méthode, tome 5, L'bumanité de l'bumanité. L'identité bumaine, Paris, Seuil, 2001, p. 345. 
L'îléité, c'est un vécu, une expérience, un ressenti particulier. Elle est teintée d'une forte affectivité qui ne peut qu'étonner tout élément extérieur à l'espace insulaire : "Qui n'a pas compris l'attachement déraisonnable des Corses pour leur île, et tellement fou qu'il apporte raison et sens absolu à leur existence, ne comprendra jamais rien à la Corse $»^{21}$. Elle est ouverture de par la potentialité des échanges qui s'offrent à elle : l'île est toujours un carrefour dans un espace maritime. Elle est aussi fermeture de par la tendance qui peut être parfois la sienne à se replier sur elle-même, pour se protéger des agresseurs qui viennent de la mer.

Le concept d'îléité appelle à réfléchir à la relation avec l'environnement, dans sa définition la plus globale, à la fois écologique et humaine. Ceux qui vivent sur les îles ressentent cette nécessité de façon impérieuse, et s'éveillent peut-être plus vite que d'autres à cet impératif écologique.

L'île est un territoire, un microcosme qui permet de façon non
utopique et avec une volonté à la fois individuelle et collective de
réfléchir aux problèmes essentiels du monde. L'équilibre de la nature
en premier lieu. Avec les questions d'environnement et de production
d'énergie. Est-il utile de rappeler par exemple comment toutes les côtes
au vent des îles du Ponant reçoivent un jour ou l'autre de plein fouet
les effets des marées noires ? L'équilibre sur le plan humain ensuite.
Avec les choix d'urbanisme et de construction, avec l'effort de
tolérance nécessaire à l'égard de l'autre, qu'il soit étranger ou insulaire ${ }^{22}$.

Au cours de la seconde moitié du $\mathrm{XX}^{\mathrm{c}}$ siècle, les habitants de la Corse ont dû se mobiliser plusieurs fois afin de prendre la défense de leur terre, menacée dans son équilibre écologique. Il ne s'agissait plus alors, comme par le passé, de défendre le territoire insulaire contre des invasions, mais contre des menaces pouvant porter atteinte à l'équilibre du système biologique insulaire. À cette occasion, sont apparus dans la sphère publique, les acteurs futurs de la défense politique de l'identité corse. Pour la plupart, la lutte écologique fut le premier pas dans l'univers de l'action politique. Deux événements marquèrent la conscience collective corse : la crise qui eut lieu suite à la décision de Michel Debré, ministre du général De Gaulle, d'installer un centre d'expérimentations nucléaires dans le massif de l'Argentella au-dessus de Calvi en 1960. Le second épisode fut celui dit des «Boues rouges",

\footnotetext{
21 José Gil, cité dans Ange Casta (dir.), La parabole corse, rencontres avec liddentité, Ajaccio, Éditions Albiana, 1995, p. 10.

22 Françoise Sylvestre, "L'âme vagabonde... ", Chemins d'étoiles, n 12, op. sit., p. 11.
} 
en 1975, suite au déversement par la société Montedison de ses déchets d'activité au large du Cap Corse, dans la mer Tyrrhénienne.

Le 13 avril 1960, le gouvernement français de l'époque annonce son intention d'installer un centre d'expérimentation nucléaire souterrain dans les mines désaffectées du Massif de l'Argentella, près de Calvi. Comme il pouvait s'y attendre, le projet donne naissance à une certaine contestation. Il est d'autant plus mal accueilli que le gouvernement doit déjà faire face à la contestation politique et sociale des insulaires depuis qu'il a décidé de supprimer la subvention qui faisait vivre les lignes du chemin de fer corse entre Bastia et Ajaccio. Or, alors qu'il vient de diminuer les crédits alloués au Programme d'Aide Régionale (PAR), le gouvernement annonce que le développement de la Corse va s'appuyer sur la militarisation de l'île : elle devra accueillir la Légion Étrangère (à Calvi et Bonifacio) ainsi qu'un centre d'essais nucléaires qui prendra la suite des expérimentations faites dans le Sahara algérien, à Reggane (décolonisation oblige). La décision du gouvernement remobilise $\mathrm{e}^{23}$

23. Le 23 octobre 1959, le gouvernement supprime dans son budget une subvention destinée au chemin de fer corse. Cette économie budgétaire aurait pu avoir pour conséquence la disparition du chemin de fer corse. Or, dans un pays montagneux et enclavé, où les routes s'apparentent plus à des chemins, cette décision aurait pu avoir des effets désastreux sur l'économie de la Corse et provoquer un sur-enclavement. Cela parait d'autant plus choquant aux Corses qui vont se mobiliser pour la défense du chemin de fer que le gouvernement semblait prêt à faire entrer la Corse dans la modernité, à travers la mise en place de la SOMIVAC en 1957, de la SETCO, du PAR et d'un plan de développement touristique. Supprimer le chemin de fer pouvait alors être interprété comme une volonté de maintenir l'île dans ses archaïsmes. Les gens vont être déconcertés par cette décision qui va à l'encontre des intérêts des structures mises en place : comment assurer un développement touristique sans infrastructures de transports adaptées ? Comment mettre en valeur un territoire, en supprimant cela même qui peut limiter l'isolement de l'intérieur des terres? La réaction des insulaires découle ainsi de leur incompréhension et de leur déception. Le mouvement de protestation et de mobilisation qui s'organise va marquer le début de la revendication politique régionaliste en Corse. Il va inaugurer l'ère des mouvements sociaux spécifiquement insulaires. Certes, il ne repose pas sur une volonté explicite de défense de l'identité corse mais, par contre, il met au centre des débats (et pour longtemps, puisque ce discours se retrouve encore aujourd'hui) la question du développement de l'île : «De 1959 date la prise de conscience des Corses. En marquant leur volonté de voir enfin l'île mise en valeur et en affirmant leur désir de combattre dans ce but, les Corses amorcent la virage. Tout ce qui surviendra par la suite découlera directement de cette prise de conscience " (Paul Silvani, Corse des années ardentes (1939-1976), Paris, Éditions Albatros, 1976, p. 106). 
contre lui des acteurs politiques et socioprofessionnels outrés par ce qu'ils considèrent une atteinte volontaire au développement économique et touristique de l'île et, bien sûr, au territoire, à la terre sacrée des ancêtres. Les insulaires ont alors l'impression d'une provocation, voire d'une volonté délibérée d'abandon de la part du gouvernement français.

On peut considérer que la mobilisation des insulaires contre l'installation d'un centre d'expérimentations nucléaires dans le massif de l'Argentella est l'une des actions collectives qui ont fondé l'émergence de la défense de l'identité corse, à travers la défense de l'intégrité de son territoire, dans l'espace public et politique en Corse. On a vu réapparaitre à ce moment-là certains des symboles de la conscience collective corse : "pour la première fois depuis des décennies, on a vu ressurgir dans les cortèges le drapeau à tête de Maure, on invoquait Pasquale Paoli, le héros de la Corse indépendante ${ }^{24}$, se souvient Edmond Siméoni, pour qui ce fut le premier combat politique. La prise de conscience du retard économique et social de la Corse a favorisé la naissance d'un sentiment d'injustice qui a $\mathrm{pu}$, par la suite, assurer le succès de la revendication régionaliste et autonomiste auprès des plus jeunes générations de Corses. Ce fut le cas pour le leader autonomiste Edmond Siméoni qui estime que ce sentiment d'injustice $^{25}$ a eu une place très importante dans son engagement personnel.

Le 3 mai 1960, une manifestation rassemble 10000 personnes à Ajaccio à l'appel d'un «comité local d'opposition à l'Argentella ». La veille, de nombreux élus s'étaient réunis à Ponte-Novo, lieu hautement symbolique puisqu'il vit la défaite de l'armée corse face aux troupes françaises, en 1769. Ici encore, identité et territoire se rencontrent : si Ponte-Novo a vu la fin historique de l'éphémère nation corse ${ }^{26}$,

24 Edmond Siméoni, Un combat pour la Corse, entretiens avec Pierre Dottelonde, op. cit., p. 33.

25 La dimension émotionnelle est omniprésente dans l'engagement d'Edmond Siméoni. Ainsi Dans un combat pour la Corse, livre d'entretien avec Pierre Dottelonde, on ne peut que remarquer la récurrence des termes "sentiment d'injustice », « juste/injuste », " épris de justice ", « injustifié », « inéquitable ».

26 Le 15 mai 1768, fut conclu le Traité de Versailles par lequel Gênes, confrontée à d'énormes difficultés financières, céda à la France la souveraineté de la Corse en échange de certains paiements. Mais il ne s'agit pas d'une simple vente : la Sérénissime prévoyait récupérer l'île dès qu'elle le pourrait. Mais la France désavoua plus tard cette partie de l'accord. Toutefois ce traité a été conclu en occultant totalement l'existence de l'État national mis en place par Paoli et la nation corse. En 
l'Argentella pourrait voir la fin du territoire corse. N'oublions pas que les catastrophes de Hiroshima et Nagasaki ne datent alors que de quinze ans, et que le nucléaire porte donc une connotation mortifère qui ne peut alors qu'entrer en résonance avec la crainte croissante d'une disparition du peuple corse.

Face au soulèvement général de l'île, Michel Debré annonce, très rapidement, le 12 mai, le retrait du projet. "Ce fut une première et grande victoire pour la communauté corse toute entière de l'intérieur et de l'extérieur ${ }^{27}$. Mais cet épisode va laisser des traces dans la société insulaire puisqu'il fait naitre un sentiment non plus seulement d'injustice, mais de méfiance durable à l'encontre des gouvernements de la France, qui nourrit la mal-compréhension que nous avons déjà évoquée entre la Corse et le continent.

À l'occasion d'un autre événement de nature écologique, vont se former ou reformer des collectifs dont certains membres ont déjà participé aux mobilisations des années soixante et dont l'engagement va se radicaliser. Il s'agit de l'affaire des « Boues rouges ». En 1969, la Montedison, multinationale spécialisée dans l'industrie chimique, décide

juillet 1768, les premières troupes françaises occupent le Cap Corse. En août arrivent des renforts, face à la résistance des paolistes qui connaissent une victoire militaire à Borgo en octobre de la même année. Au printemps 1769, 20000 militaires français débarquent. Le 8 mai 1769, à Ponte-Novo, les troupes corses sont écrasées par l'armée française. La Corse entre définitivement dans le giron français. Le 13 juillet, Pascal Paoli quitte la Corse pour l'exil. Depuis de nombreuses années, les militants nationalistes, de tous bords, se réunissent pour commémorer cette défaite, dans une cérémonie qui parfois constitue une véritable trêve lors des affrontements des groupes rivaux. Les militants ne remettent pas en cause cette défaite, ils l'acceptent en tant que telle. Alors pourquoi la commémorer? L'hypothèse de Delphine Tillard peut sembler surprenante à qui ne connaît pas l'île, mais reste tout à fait probable si on se place dans l'univers des représentations corses : «La logique de ce souvenir se situe en fait ici dans un tout autre registre : elle s'inscrit dans le champ du juridique traditionnel insulaire, à savoir celui de la vendetta. [...] L'affrontement de Ponte-Novo rappelle ainsi qu'une nation (française) en a écrasé une autre (la Corse alors indépendante), acte éminemment réprouvé par la morale corse. Il n'est alors pas vu comme une défaite en soi mais comme l'emblème de toutes les atteintes à l'honneur de l'île, la première et la plus grave » (Delphine Tillard, «La Bataille de Ponte-Novo. Liturgie d'une défaite? ", Socio-anthropologie, $\mathrm{n}^{\circ}$ 9, Commémorer, consulté sur le site : http://revel.unice.fr/anthropo/document.html?id=10).

27 Edmond Siméoni, Un combat pour la Corse..., op. cit., p. 34. 
de construire une usine de production de bioxyde de titane en Toscane. Cette activité est très polluante. Les déchets qui en sont issus constituent les boues rouges qui sont composées, entre autres, d'acide sulfurique, de chrome, d'oxyde de fer, d'oxyde manganèse, etc. En 1971, l'usine de Scarlino est achevée. Mais elle ne comporte pas d'épurateur, la société ayant jugé plus rentable d'immerger les boues polluantes dans la mer Tyrrhénienne, c'est-à-dire au large du Cap Corse, dans un lieu, le Bas Fonds des Veuves, où de nombreux courants marins se rencontrent ${ }^{28}$.

Le journaliste Aimé Pietri informe les Corses dans un article paru dans le journal Le Provençal, le 29 avril 1972. Un premier comité antiboues rouges ${ }^{29}$ se met en place à Bastia. D'autres suivront à Ajaccio et sur le continent, à Marseille, à Nice et dans d'autres ports français de la Méditerranée, eux aussi menacés par cette pollution. Les élus de HauteCorse commencent à interpeller les pouvoirs publics, un mois après l'article d'Aimé Pietri. Le Conseil Général, présidé par François Giacobbi, demande une enquête. Jean Zuccarelli, maire de Bastia, et son conseil municipal demandent aux ministres des Affaires étrangères et de la Marine Marchande d'intervenir auprès de l'Italie. Face à l'inertie des pouvoirs publics, une manifestation est organisée le 17 février 1973, à l'appel de tous les comités de l'île. Elle entend jouer sur la symbolique et le caractère solidaire de la contestation écolo-identitaire, sa filiation avec ce qui s'est passé quelques années auparavant : "Le tocsin commença à sonner. Je l'avais demandé au vieux chanoine Mattei, me souvenant que les Calvais avaient défilé au son du tocsin lors du tollé général qu'avait suscité en Corse le projet du gouvernement de faire de

28 Dès avant la construction de l'usine, les riverains s'inquiètent et un premier rapport du Laboratoire d'Hydrobiologie de Rome confirme les conséquences mortelles de ces déversements pour l'ensemble de l'écosystème maritime. Mais, sur autorisation du Ministère italien de la Marine Marchande, les boues sont rejetées de façon expérimentale. Un nouveau rapport du même institut romain vient nuancer les premières conclusions. Alors que le Ministère décide dans un premier temps d'interdire, en novembre 1971, les déversements, il accorde finalement en mai 1972 une autorisation provisoire d'immersion qui sera reconduite plusieurs fois. Le commandant du port de Livourne essaye d'empêcher cette agression contre l'environnement mais il n'a d'autre choix que de céder face aux ordres de son ministère.

29 Lucia Molinelli-Cancellieri, Boues Rouges, la Corse dit non, Paris, L'Harmattan, 1995. Cet ouvrage retrace toute l'histoire de ce mouvement collectif. 
l'Argentella une poubelle nucléaire $»^{30}$. On voit également le drapeau dans le défilé de même que résonne l'hymne corse. L'affirmation que la défense de l'identité collective corse et celle du territoire sont indissociables est donc faite de manière certes symbolique mais également explicite.

Mais la manifestation dégénère : la sous-préfecture est prise d'assaut par une centaine de jeunes manifestants malgré les appels au calme d'Edmond Siméoni. Les Compagnies républicaines de sécurité (CRS) interviennent, mais le sous-préfet est quelque peu molesté par certains manifestants. De nombreux blessés sont comptabilisés de part et d'autre des deux camps. De nombreuses arrestations ont lieu, dont celle de Vincent Duriani, adjoint communiste du maire de Bastia et celle d'Edmond Siméoni. Ceci va raviver les tensions à Bastia, d'autant que la situation écologique ne s'améliore pas. Le 26 février 1973 a lieu l'opération Isula Morta (Île morte) qui est très suivie. La journée se termine positivement puisque les deux inculpés sont relâchés, bien que les charges contre eux ne soient toujours pas abandonnées.

S'il est clair qu'il y a une prise de conscience collective de la part des insulaires des difficultés qui sont les leurs, elle s'accompagne d'un sentiment d'injustice qui cède la place peu à peu à la colère. L'idée que les relations entre l'État et les Corses qui veulent protéger leur terre ne peuvent que se durcir s'impose chez certains sympathisants de la cause écologique. "Ce mouvement de protection du milieu introduira indirectement l'utilisation de la violence comme moyen d'expression contestataire; moyen dont le recours sera en progression constante ${ }^{31}$.

La mobilisation est forte également chez les jeunes Corses du continent : à Marseille, des étudiants et universitaires, parmi lesquels ont trouve Paul Patriarche, futur vice-président de l'Assemblée de Corse, font une grève de la faim pour dénoncer l'inertie du gouvernement malgré les conclusions du rapport Aubert qui confirme la nocivité des rejets de la Montedison. D'autres manifestations ont eu lieu un peu partout en Méditerranée. La Montedison essaye de composer avec les mouvements de protestation et fait ses déversements plus profondément pour diluer la pollution. Mais cela n'a que peu d'impact sur la situation

Lucia Molinelli-Cancelliere, Boues Rouges..., op. cit., p. 39.

31 Claude Olivesi, « La Corse contestataire, l'État et l'Europe », dans Fred Reno (dir.), Identité et politique. De la Caraibe et de l'Europe multiculturelles, Paris, Economica, Caraibe - Amérique Latine, 1995, p. 162. 
environnementale. La revendication va connaittre de nombreux accès de violence par la suite : en septembre 1973, des attentats à l'explosif ont lieu contre des navires à Bastia et Livourne. Toutefois l'affaire des Boues rouges n'est pas réglée pour autant. Un procès sera finalement ouvert en Italie en 1974, contre la Montedison. L'épilogue n'aura lieu qu'en 1984 avec l'arrêt des déversements des boues polluantes en Méditerranée.

Cette période marque le début de l'engagement militant pour beaucoup de jeunes Corses. L'affaire des Boues rouges a favorisé l'essor du mouvement autonomiste ${ }^{32}$, car le territoire insulaire a été menacé une fois de plus, une fois de trop pourrait-on dire pour ceux qui étaient déjà engagés dans une contestation de l'action des gouvernements français en Corse.

Le climat était en train de changer, les petits signes d'une remise en cause de la France avaient commencé à se multiplier. Dans ce contexte, il est certain que l'affaire des boues rouges a joué un rôle considérable dans la dégradation de l'image de la France en Corse. C'était une affaire d'une gravité extrême parce qu'elle menaçait notre île sur le long terme. [...] De manière générale, les Corses ont ressenti de manière presque psychanalytique cette agression contre leur environnement, leur terre, leur mère, la mer Méditerranée ${ }^{33}$.

Ces événements ont fait émerger la question du modèle de développement à envisager pour la Corse. Les Corses devaient-ils subir un modèle imposé par l'État au risque de voir leur terre et leur identité menacées? Une solution ne pouvait-elle naitre qui corresponde aux attentes des insulaires et qui les inscrivent dans une modernité qui les amènerait à rattraper les retards de l'île en matière d'infrastructures, tout en protégeant l'intégrité de l'île ? Pour certains acteurs des mouvements que nous venons d'évoquer rapidement, l'autonomie politique apparaît comme cette solution ${ }^{34}$.

32 Pour plus de détails sur l'histoire de ce mouvement, voir, entres autres, Emmanuel Bernabeu-Casanova, Le nationalisme corse : genèse, succès et échec, Paris, L'Harmattan, 1997 ; Xavier Crettiez, La question corse, op. cit.

33 Edmond Siméoni, Un combat pour la Corse..., op. cit., p. 70.

34 C'est en juillet 1973 que le mouvement autonomiste nait véritablement sur la scène politique corse, lors de l'assemblée générale qui va transformer l'Action Régionaliste Corse (créée en 1967) en Action pour la Renaissance de la Corse (Azzione per a Rinascita di a Corsica). On passe donc d'une revendication régionaliste, fondée sur une volonté de développement économique et de reconnaissance de l’identité corse 
Aujourd'hui, la réflexion sur l'autonomie ${ }^{35}$ de la Corse a mûri. Elle s'ancre dans une démarche qui s'est construite au fil des ans, en prenant en compte des expériences proches comme celles de la Sardaigne que les élus corses citent souvent en exemple. En effet, cette île sœur nourrit l'imaginaire de nombreux hommes politiques. Pour José Rossi, président de l'Assemblée de Corse de 1998 à 2002, « [1] Sardaigne est, naturellement, l'île sœur de la Corse. [...] Je pense qu'il est tout à fait opportun d'aller plus vite et plus loin afin de créer un véritable climat affectif entre les Sardes et les Corses que tout rapproche $»^{36}$.

à une revendication plus précise, fondée sur un projet politique. La démarche était à l'époque osée, comme le rappelle Edmond Siméoni : "Le mot d'autonomie était connoté d'indépendance, mais également d'irrédentisme: Et des exemples comme la Sardaigne, qui jouit d'un statut d'autonomie depuis 1947, étaient inutilisables précisément à cause de l'irrédentisme auquel avait adhéré une poignée de Corses à la fin des années 30. Il fallait donc une thérapie progressive, expliquer aux Corses que l'autonomie interne était quelque chose de souhaitable, que c'était une forme d'appartenance, même si l'idée représentait à l'époque une révolution majeure des mentalités. Notre position a été très bien résumée à ce moment dans Arriti sous la plume de "Tragulinu" : "L'autonomie a l'avantage de concilier la survie de notre ethnie [comprenez peuple] et son appartenance à la communauté française, l'aspiration légitime et le réalisme" " (Edmond Siméoni, Un combat pour la Corse..., op. cit., p. 71). Nous ne pouvons pas développer plus longuement ici la question de l'autonomisme et de l'indépendantisme en Corse.

35 L'autonomic est un moyen pour un État de préserver son unité tout en déléguant des compétences déterminées à une partie de sa population à qui il reconnaît un caractère spécifique au sein de la communauté nationale. Le type d'autonomie qui nous intéresse ici est l'autonomie politique territoriale : c'est « un ensemble de mesures qui visent à garantir un certain degré d'auto-identification à un groupe qui diffère de la majorité de la population de l'État mais qui est majoritaire dans une région donnée. L'autonomie implique une répartition des compétences entre les autorités centrales et l'entité autonome. Les compétences de l'entité portent généralement sur les affaires culturelles, économiques et sociales. [...] Par sa nature même, l'autonomie requiert une certaine coopération et une certaine coordination entre les autorités centrales et locales » (Ruth Lapidoth, "Les caractéristiques de l'autonomie ", dans Centre Européen des questions de minorités, Autonomies insulaires, Ajaccio, Éditions Albiana, 1999, p. 27).

36 Emmanuel Bernabeu-Casanova et Dominique Lanzalavi Dominique, Corse, les voies de lavenir, Paris, L'Harmattan, 2003, p. 91-92. La Sardaigne est autonome depuis 1945 : elle possède un droit d'initiative dans plusieurs domaines législatifs comme tout ce qui touche aux énergies, au commerce, à l'agriculture, à la pêche, aux travaux publics, à la police locale, aux services administratifs. La proximité entre les deux îles est plus que géographique, elle est historique (elles ont longtemps fait partie du même royaume), culturelle, humaine. 
Mais les regards des porteurs de ce projet politique se tournent également vers l'Union Européenne. Car, dans le cadre du traité d'Amsterdam de 1997, les pays de l'Union Européenne reconnaissent aux îles le caractère de régions spécifiques qui doivent faire face aux problèmes posés par leur insularité.

Les îles sont des régions avec des traits particuliers : elles sont souvent marquées par la « super-périphéralité »; un grand retard de développement économique par rapport aux régions continentales; et les handicaps dus à l'insularité qui cause de graves problèmes dans le domaine des transports ainsi qu'en matière d'importation et d'exportation. En outre, les îles sont très souvent dépendantes des États pour les subventions nécessaires à leur survie. [...] Cependant les îles-régions possèdent aussi des atouts. Leur qualité de vie et leur cadre naturel est souvent supérieur à ceux des grandes villes (où résident $80 \%$ des européens). Elles gardent plus longtemps une culture spécifique - qui peut être linguistique - ou simplement un mode de vie et un ensemble de coutumes ${ }^{37}$.

Ce bref rappel historique et géopolitique nous a permis de voir la force du lien des Corses à leur territoire et de concrétiser ce concept d'îléité. Cette dernière repose sur un vécu dialogique en ce sens où le lien si fort, comme nous venons de le voir, qui unit l'îlien à sa terre peut être la source même de son enfermement dans sa matrice originelle, mais, en même temps, l'îlien ne peut pas exister sans la mer ou l'océan. L'ouverture sur l'extérieur est donc nécessaire à l'îlien pour qu'il connaisse l'expérience de l'altérité et de la différence. Cette dualité de l'identité insulaire est fort bien décrite par le philosophe corse JeanToussaint Desanti.

Unifiés par la mer qui désignait nos bornes, nous avons été divisés par la terre, singularisés à l'extrême. Ce petit monde si bien dessiné, qu'une vue aérienne nous livre aujourd'hui en son entier, a été formé de mondes multiples et séparés. Au milieu du siècle dernier encore, la Balagna était un autre monde pour un habitant du Sartenais. Simples données ponctuelles, mais qui comportent leur poids symbolique : la terre habitée, le sol qui porte les pas et où sont couchés les morts, la terre refuge nourricier, la terre, toujours la terre, irremplaçable en sa singularité. Notre insularité était-elle donc aussi contradictoire? La borne maritime qui nous unifiait était-elle donc à ce point inquiétante qu'elle nous divisait tout autant, nous assignant à ces lieux de naissance

37 John Loughlin, «Introduction », dans Centre Européen des questions de minorités, Autonomies insulaires, op. cit., p. 15. 


\section{NPSS, VOLUME 2, NUMÉRO 1, 2006}

et de clôture que furent nos vallées et nos pievi ? Oui, il en fut ainsi pour nous, je crois ${ }^{38}$.

L'îléité est une relation particulière avec un territoire, car la frontière entre Soi et l'Autre est ici matérielle. Elle va donner un cadre à l'identité. L'îlien est dans et par son île. "On porte l'île avec soi. C'est l'ubiquité du corps insulaire, qui est à la fois là où il est et ailleurs. Lorsqu'on est en Corse, on est ailleurs. Lorsqu'on est ailleurs, on est en Corse. [...] Il faut apprendre à penser cette dualité du corps insulaire ${ }^{39}$. Et l'Autre est au-delà de la mer. La frontière avec l'Autre est donc physiquement identifiable. Mais, pour autant, comme toute identité, l'identité îlienne ne peut se contenter ni d'elle-même, ni de cette distance. Pour perdurer, elle doit nécessairement se confronter à l'altérité.

\subsubsection{La nécessaire relation à l'Autre}

Le fait de vivre sur une île a des conséquences sur la façon d'appréhender la relation à la vie et à l'Autre, surtout dans les moments où cet Autre est moins présent.

La vie sur une île est simple. La nature est belle, forte, suffisante. L'horizon est infini, source de rêve et d'inspiration. On se contente de ce qu'il y a en matière de biens de consommation, de services et de culture $^{41}$. Et précisément parce qu'ils sont limités, on s'en nourrit le mieux et le plus possible. En outre, comme la plupart des îles sont tournées vers le tourisme, on élargit le champ de ses relations avec les autres. On leur apporte. Ils nous apportent. Les périodes où il n'y a personne et celles où il y a trop de monde sont chacune à leur manière enrichissantes et sources de réflexion. Leur alternance rythme la vie et l'organisation de l'île ${ }^{41}$.

38 Jean-Toussaint Desanti, «Effacer la mer. Une réflexion sur l'identité corse », Esprit, Dossier La Fièvre identitaire, janvier 1997, p. 151.

39 Jean-Toussaint Desanti, «Les lieux sont tombés sur la tête », dans Ange Casta (dir.), La parabole corse, rencontres avec l'identité, Ajaccio, Éditions Albiana, 1995, p. 11.

4i) Ce constat est celui de Françoise Sylvestre. Nous verrons par la suite que tous, et notamment les élus que nous avons rencontrés, ne se contentent de ce minimum et en font une revendication pour l'action en Corse : « remise à niveau », « rattrapage ».

41 Françoise Sylvestre, «L'âme vagabonde, d'une île à l'autre », Chemins d'étoile, n ${ }^{\circ} 12$, op. cit., p. 10-11. 
La question de l'altérité dans la culture et la société corses nous semble avoir évolué depuis les années quatre-vingt-dix, c'est-à-dire à partir du moment où la question du peuple corse et de son devenir est entrée un peu plus dans le débat public en France. En 1990, Claude Orsoni ${ }^{42}$ évoquait la question de l'altérité dans la culture corse en considérant que l'histoire de la Corse l'avait amené à une fermeture à l'Autre. Toutefois, il évoquait la possibilité d'une ouverture, qui possède aujourd'hui une résonance particulière, quasi prophétique, au vu de l'évolution de la conjoncture politique en Corse.

Cette situation ne semble pouvoir évoluer que par la transformation de la société corse en une communauté assez autonome et dynamisée pour ne plus redouter la présence des autres communautés et leur interférence dans le rapport à l'État ; ce qui signifie aussi : par l'engagement des individus dans des entreprises ouvertes sur tous les horizons du monde extérieur, à commencer par des horizons méditerranćens ; pour une telle évolution, le cadre le plus favorable serait peut-être davantage méditerranéen et européen que français ${ }^{43}$.

Le personnel politique actuel semble intégrer, bon gré, mal gré, cette attitude plus ouverte à l'Autre, à travers, conformément aux attentes évoquées par Claude Orsoni, des liens tissés en Méditerranée et en Europe. Le programme IMEDOC ${ }^{44}$ en est une illustration importante.

42 Claude Orsoni, «Clanisme et racisme. Hypothèses sur les relations inter-communautaires en Corse ", Peuples Méditerranéens, $n^{\circ}$ 51, Le néo-racisme en Corse, avril-juin 1990, p. 191-201.

43 Ibid., p. 200.

${ }_{44}$ Créé le 9 mai 1995, le réseau IMEDOC (Îles de la Méditerranée Occidentale) regroupe des îles de la Méditerranée (Les Baléares, la Corse, la Sardaigne, la Sicile depuis le 17 avril 2000). Son objectif principal était de créer un « front commun des îles de la Méditerranée ». L'intitulé peut sembler un peu vindicatif en ce sens où il pourrait laisser sous-entendre que ces îles seraient attaqués on ne sait par quel ennemi, ou du moins elles devraient se défendre devant une quelconque adversité (sous-entendu continentale). Se trouve là un ressenti insulaire que nous rencontrerons de façon récurrente au cours de ce travail. En fait, il s'agit d'une association d'îles dont les préoccupations peuvent être proches et dont les dirigeants ont souhaité s'associer pour mutualiser leurs ressources et monter ainsi un certain nombre de projets que chacune n'aurait pu réaliser seule et mettre en œuvre une «collaboration institutionnelle permanente entre les îles pour la défense de leurs particularités insulaires ». Ce réseau s'est depuis développé puisque la Crête avait fait la demande d'intégrer IMEDOC. Aussi un nouvel accord de coopération insulaire, 
Mais d'autres types d'actions et de rencontres sont réalisés également. À la fois pour que la Corse puisse être reconnue en tant qu'entité réelle, mais aussi pour chercher des solutions pour l'avenir.

Aussi, dans le projet politique des militants nationalistes tel qu'il nous fut présenté par Jean-Guy Talamoni ${ }^{45}$, la relation à l'Autre est considérée comme nécessaire puisque favorisant la reconnaissance de la Corse comme une entité ayant une certaine existence au niveau international (avant d'avoir une reconnaissance en tant qu'entité politique autonome ou indépendante).

Je crois que la Corse doit à un moment donné être une entité [...] reconnue au plan international, alors bien entendu nous avons des idées très précises là-dessus, mais en tous cas, il est vrai que la Corse commence à avoir une manière d'être représentée au plan international à travers des démarches para-diplomatiques. C'est vrai que la Corse se déplace notamment en Méditerranée avec IMEDOC, etc. Nous, nous voyons d'un bon oil toutes ces démarches qui visent à présenter la Corse comme une entité culturelle, économique et politique.

Le fait que les acteurs politiques corses aient souhaité se rapprocher des autres îles de la Méditerranée occidentale, bien que reposant sur une proximité géographique, s'explique également parce que chacune a connu l'expérience du rattachement à un espace continental, pas toujours choisi et souvent justifié par l'importance stratégique relative de chacune dans l'espace maritime. La relation à l'Autre repose alors sur l'idée d'un ressenti commun.

[La Corse] appartient [...] au monde insulaire ouest-Méditerranéen mais l'unité de cet ensemble n'a jamais réellement existé, dans la mesure où ces îles ont été dépendantes de pouvoirs continentaux différents. La similitude réside probablement en un sentiment de voisinage avec l'impression d'« avoir vécu la même chose » et d'exprimer des revendications très proches : insularisme évoluant idéologiquement en autonomisme... ${ }^{46}$

«Eurimed-Îles de la Méditerranée » a-t-il été signé en octobre 2004, à Taormina, en Sicile, entre les Baléares, la Corse, la Crête, la Sardaigne et la Sicile. IMEDOC s'est donc de ce fait ouvert aux îles de la Méditerranée Orientale, tout en conservant les mêmes objectifs de sensibilisation aux spécificités insulaires et méditerranéennes, à la coopération transrégionale dans les domaines économiques, sociaux et culturels.

${ }^{45}$ Élu du groupe Corsica Nazione à l'Assemblée Territoriale de Corse.

46 Joseph Martinetti, Insularité et marginalité..., op. cit, p. 41. 
Ces projections dans l'espace (l'environnement) et le temps alimentent un certain nombre de représentations qui peuvent être partagées par les îliens et qui sont en perpétuelles reconstruction et réorganisation.

\subsubsection{Réinventer le territoire insulaire}

"Confrontés à l'espace connu, dont pourtant les traits nous sont familiers, il nous arrive de ressentir une émotion indéfinissable, l'intuition d'une vérité voilée qui reste à découvrir... » ${ }^{47}$. Vivre sur un territoire est producteur à la fois de sens, d'inventivité, d'imaginaire : "L'être, singulier ou collectif, qui vit en harmonie avec son territoire dessine les contours d'une géographie poḯtique. L'être heureux est sans doute celui qui, comme l'enfant, ici et maintenant, s'abandonne à une lecture inventive des formes familières et quotidiennes de son jardin, pour les métamorphoser en un monde nouveau, apprivoisé où tout se transforme sans pour autant jamais se dévoiler complètement ${ }^{48}$.

Que faut-il comprendre par là? Qu'habiter un territoire, en l'occurrence ici un territoire insulaire, alimente un imaginaire qui va construire et justifier le lien que les acteurs peuvent vivre avec ce territoire. Mais cela signifie aussi que cet imaginaire alimentera des représentations plus globales sur le territoire concerné.

D'une certaine manière, tout territoire social est un phénomène immatériel et symbolique, [...] tout élément même physique ou biologique n'entre dans la composition d'un territoire qu'après être passé par le crible d'un processus de symbolisation qui le dématérialise en quelque sorte. Tout territoire social est un produit de l'imaginaire humain ${ }^{49}$.

\subsubsection{L'imaginaire insulaire}

Prendre en compte l'imaginaire insulaire, c'est-à-dire l'ensemble des activités symboliques qui permettent de construire et d'alimenter le lien

47 Michel Roux, Le Ré-enchantement du territoire, publié en mars 2001 sur le site http://www.mcxapc.org/, consulté en mars 2003.

48 Ibid.

49 Yves Barel, « Le social et ses territoires », dans François Auriac et Roger Brunet (dir.), Espaces, jeux et enjeux, Paris, Fayard, 1986, cité par Philippe Tizon, «Qu'est-ce que le territoire? ", dans Guy Di Meo (dir.), Les territoires du quotidien, Paris, L'Harmattan, Géographie sociale, 1996, p. 21. 
au territoire, apparaît ici nécessaire pour comprendre la relation des Corses à leur île et le rôle qu'elle joue dans la formation de leur identité collective. Nous partageons ici l'opinion de Dominique Verdoni quand elle écrit :

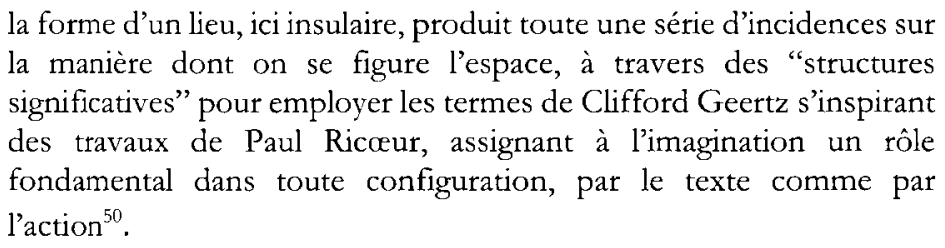

Nous avons vu précédemment que le concept d'îléité intègre le vécu des insulaires. Or, pour les îliens, "l'image dominante de l'île est celle de la terre : leur terre-patrimoine qu'ils ont tant de mal à partager $n^{51}$. Le philosophe corse Jean-Toussaint Desanti évoque son expérience personnelle et la nécessité pour les insulaires "d'effacer la mer ${ }^{52}$ pour rencontrer l'Autre qui est au-delà. Mais l'île est également présente dans l'univers des représentations des non insulaires : «pour les continentaux, l'image dominante de l'île est d'abord l'expérience de la mer à franchir comme une épreuve, la première phase de l'initiation, la première étape d'une appropriation réelle ou supposée, vécue ou fantasmée " ${ }^{53}$.

L'espace insulaire possède un fort impact symbolique : il est source de mythes, qui vont non seulement participer de la construction de l'identité collective corse, mais aussi de toutes les identités insulaires. Toutefois, il serait faux de considérer que cet imaginaire insulaire est unique. Ainsi, les îles du Pacifique Sud, par exemple, n'éveillent pas les mêmes sentiments que les îles de la Méditerranée ou de l'Atlantique chez les navigateurs de l'époque des Grandes Découvertes ${ }^{54}$.

50) Dominique Verdoni, "L'insularité : approche herméneutique de la variation ", dans Anne Meistersheim (dir.), L'île laboratoire, op. cit., p. 437.

51 Anne Meistersheim, Figures de l'ile, op. cit., p. 70.

52 Jean-Toussaint Desanti, «Effacer la mer. Une réflexion sur l'identité corse », op. cit., p. $148-155$.

53 Anne Meistersheim, Figures de l'ile, op. cit., p. 70.

54 Christian Huetz de Lemps, "Des hommes et des îles : l'originalité du Pacifique ", Chemins d'toiles $\mathrm{n}^{\circ} 12$, op. cit. 
Mais aborder la question de l'imaginaire insulaire appelle à sortir des références classiques des sciences sociales et humaines pour nous intéresser un moment aux travaux existant dans le domaine littéraire sur le monde des îles. Nous nous référerons notamment à l'ouvrage Impressions d'îles ${ }^{55}$, dont les textes ont été réunis par Françoise Létoublon, mais également à des extraits d'œuvres littéraires. Bien sûr, nous ne chercherons pas ici à aborder l'ensemble de la littérature mais à voir en quoi s'intéresser à des territoires insulaires n'est pas anodin, ni scientifiquement, ni intellectuellement, ni même émotionnellement puisque les îles sont liées plus ou moins, d'une manière ou d'une autre, à l'imaginaire, individuel ou collectif.

L'île a très largement alimenté l'imaginaire des écrivains, et ce, depuis des siècles, voire des millénaires : «le thème de l'île semble se rencontrer sitôt que la littérature existe, et avant même qu'elle ne soit conservée sous forme écrite, dans les mythologies " ${ }^{56}$. C'est dans les mythologies grecques que l'on trouve le plus de références aux îles : «De nombreux mythes cherchent à rendre compte de l'existence des îles et de leurs particularités : les mythes grecs de ce type sont particulièrement nombreux, ce qui s'explique peut-être par le nombre des îles grecques et leur variété réelle $»^{57}$. Les îles servent également de théâtre aux aventures et aux voyages, comme, bien sûr, celui d'Ulysse. Elles servent également à évoquer les origines des cités grecques à travers les mythes d'installation ou de fondation ${ }^{58}$.

Mais l'île n'est pas l'apanage de la mythologie grecque. La littérature européenne s'y est également abondamment référée. Outre Rabelais ou Dante, on ne peut pas ne pas évoquer Thomas More et son Utopia, isthme devenu île par la volonté de son roi Utopus qui y instaure un système politique démocratique, parlementaire, et dont l'organisation est fondée sur la famille.

\footnotetext{
55 Françoise Letoublon (dir.), Impressions d'îles, Toulouse, Presses Universitaires du Mirail, 1996.

56 Françoise Letoublon, Paola Ceccarelli et Jean Sgard Jean, "Qu'est-ce qu'une île? ”, dans Françoise Letoublon (dir.), Impressions d'îles, op. cit., p. 10.

57 Ibid., p. 16.

58 Ibid., p. 22-23.
} 
Dans l'ensemble de la littérature, on navigue alors d'une île Éden à une île enfer (qui peut oublier Robinson isolé sur son île ?), en passant par l'île-purgatoire de Dante ou l'île ambiguë comme la Vezzano de Julien Gracq dans le Rivage des Syrtes. Malheureusement nous ne pouvons évoquer ici toutes ces figures mythiques de l'île tant la littérature en foisonne, car ces mythes sont bien vivants comme nous le rappelle Françoise Létoublon:

Cette tendance des îles à fabriquer du mythe est-elle morte avec le grec, le latin de Thomas More et le français de Rabelais ? Les récits de Calvino sur le folklore de Sicile, ceux de Marguerite Yourcenar sur les îles grecques dans lesquelles elle a vécu, les légendes celtiques et germaniques, peuvent laisser croire qu'il existe encore des îles où les bons vieux mythes sont vivants : les nymphes, les Néréides et les Sirènes peuplent encore les îles. Et si le mythe se meure, l'utopie a des chances d'exister aussi longtemps que les peuples rêveront du bonheur. [...] De la description encyclopédique aux rêves les plus fous, les îles entrainnent dans leur sillage toute une floraison de textes et de réflexions : c'est une invitation à un voyage aux Antipodes, ou au moins autour d'un Atlas, et le monde entier pourrait être considéré comme une île pour l'imaginaire ${ }^{59}$.

Même si nous ne pouvons pas faire le tour de tous les aspects de la littérature abordant l'imaginaire insulaire ${ }^{60}$, on ne peut manquer de s'intéresser aux auteurs qui ont abordé l'imaginaire insulaire corse ou méditerranéen. Dans le cadre de cette recherche, nous avons privilégié quelques œuvres comme, par exemple, Le Guépard de Lampedusa, La Corse, ou encore la Maison des Viale de Paul-Michel Villa. Ces ouvrages permettent d'aborder la force que recèle tout ce qui a trait à l'île dans l'imaginaire corse. Mais, nous nous contenterons ici de les aborder «chemin faisant », pour leur valeur herméneutique.

$59 \quad$ Ibid., p. 27.

60 Outre les références bibliographiques citées ici, nous renvoyons au site Internet du festival du Livre insulaire de Ouessant (www.livre-insulaire.fr $\backslash$ index.html) qui possède une très large bibliographie des romans, recueils de poésie, essais, ouvrages scientifiques abordant la question de l'insularité. Cette bibliographie concerne toutes les zones du monde. Le festival a lieu tous les ans à la fin du mois d'août sur l'île d'Ouessant, en Bretagne (France), et consacre divers ouvrages sur l'insularité. Chaque année, le festival aborde une île ou un système d'îles particulier : Irlande, Antilles, la Méditerranée, etc. 
La dimension mythique ou mythologique ne doit pas être considérée comme une façon de déterritorialiser la dimension insulaire. Bien au contraire, en la nourrissant de symboles, de représentations, parfois convergents, parfois contradictoires ou dérangeants, l'imaginaire contribue à lui donner une matérialité certaine. Ces représentations et symboles seront autant de références nécessaires à la compréhension des problématiques insulaires, notamment dans une approche en terme d'île-système qui permet d'associer ces diverses représentations.

\subsubsection{L'île-système}

D'un point de vue méthodologique, nous avons pris le parti de faire appel à certains outils de l'analyse systémique complexe pour construire notre réflexion. Ce postulat méthodologique apparaît ici nécessaire car les différents éléments que nous avons observés et rassemblés s'imposent à nous comme devant se penser ensemble. Ce faisant, ils influent sur la façon de se représenter l'île, non pas comme un espace indépendant des autres espaces (géographiques, sociaux, etc.), mais comme un système en interrelation avec son environnement.

Le système, tel que nous le concevons ici, s'inscrit dans un projet global de connaissance.

La démarche systémique consiste toujours en effet à isoler un certain nombre d'éléments $n$, en privilégiant certains types de relations qui vont conférer à ce système une relative autonomie par rapport à un ensemble d'éléments plus vaste $\mathrm{N}$. Ce caractère global de la modélisation systémique ne doit pas être interprété comme un objectif d'exhaustivité. [...] C'est le chercheur qui décide d'inclure telle ou telle variable dans le système et d'en exclure. Ces choix s'effectuent par rapport aux idées, aux objectifs plus ou moins teintés de subjectivité du chercheur, à ses hypothèses de travail. Il appartient au chercheur dans la perspective de sa recherche de centrer son attention sur certains éléments plutôt que sur d'autres ${ }^{61}$.

La définition du système que nous retenons est celle développée par Edgar Morin : " une unité globale onganisée d'interrelations entre éléments, actions ou individus $»^{62}$. L'importance est placée ici plus sur les interrelations que sur les éléments, c'est-à-dire appliquée à l'île, ce qui

${ }_{61}$ Jean-Claude Lugan, La systémique sociale, Paris, PUF, Que sais-je ?, 1993, p. 23-24.

${ }^{62}$ Ibid., p. 29. 
compte, c'est moins le territoire ou le sentiment d'identité qui y est associé que la relation entre ce territoire et les actions des acteurs, décideurs, élus ou encore que la relation entre le territoire insulaire et d'autres territoires, ou entre l'identité collective corse et l'identité collective française, italienne, méditerranéenne, etc. Nous nous trouvons là dans une posture intellectuelle, celle de la pensée complexe développée par Edgar Morin dans les six tomes de La Méthode ${ }^{63}$ qui se veut moins réductionniste qu'une autre qui se contenterait de faire la liste des éléments constitutifs du système sans les relier entre eux.

L'une des premières qualités que l'on reconnaît à un système est celle de ses limites. S'agissant d'une île telle que la Corse, la question peut sembler quelque peu triviale. Ses limites géographiques peuvent paraître suffisantes à définir le système insulaire corse. Or, ces limites peuvent engendrer une vision réductrice du système insulaire, notamment concernant sa population. Doit-on considérer exclusivement les habitants de l'île et ignorer les membres de la diaspora, alors que ceux-ci peuvent revenir fréquemment et également posséder divers biens (maisons, commerce, etc.), ce qui rend leur lien avec l'île encore plus concret, comme nous le montre, par exemple, le fait que nombre d'exîlés continuent à voter dans le village dont ils sont originaires. Ce double visage de la population corse engendre nombre de flux, de relations qui lient l'île à un environnement très vaste.

C'est pourquoi, à partir des éléments que nous avons observés sur la Corse, sur sa relation avec la Méditerranée, avec les différents États qui y ont exercé leur souveraineté, etc., nous pouvons définir la Corse comme un système ouvert, car procédant à des échanges avec son environnement. Concrètement, ces échanges peuvent être des échanges d'information (à travers des réseaux comme IMEDOC, par exemple, où chacun échange de l'expérience, des ressources matérielles ou immatérielles) ou d'énergie (c'est ainsi que l'on pourrait voir les mouvements de population entre la Sardaigne et la Corse, ou entre la Corse et les colonies). Ils peuvent participer de la transformation du système : ainsi la coopération interrégionale avec les autres îles de la Méditerranée a des effets sur les structures économiques de l'île mais également sur les structures mentales des élus qui intègrent la dimension coopérative dans l'action politique locale, comme un moyen de

63 Edgar Morin, La Méthode, 6 tomes, Paris, Seuil, 1977-2004. 
préserver et de renforcer l'identité méditerranéenne de la Corse : "Changer pour que rien ne change... C'est-à-dire que le système ouvert vit de ses changements et qu'il maintient son identité en intégrant les différences $»^{64}$.

Nous nous trouvons ici face au principe dialogique décrit par Edgar Morin comme l'un des trois principes de base de la pensée complexe: la complexité du système insulaire repose sur l'idée que, à côté de cette ouverture sur l'environnement, existe une tendance à la fermeture sur lui-même du système insulaire, sur une volonté de conserver ce qui fait son identité.

Cette oscillation entre ouverture et fermeture, entre changement (développement ?) et clôture (maintenance, conservation, identité) est centrale dans les systèmes insulaires. Elle semble d'ailleurs tout à fait correspondre à la description de la vie insulaire faite par Fernand Braudel : « on a si souvent signalé cet archaisme des îles, leur étrange pouvoir de conserver, des siècles durant, d'antiques formes de civilisations ou le mélange de leur folklore, qu'il est inutile d'y insister longuement. Mais en même temps, à l'opposé de ce repliement, il leur arrive d'un coup, au hasard de quelque changement de maitre ou de fortune, d'accueillir tout un pan nouveau de vie ou civilisation $"{ }^{65}$.

N'est-ce pas là le fondement de la construction de l'identité corse, reposant sur un ancrage territorial et communautaire fort, mais intégrant les apports de ces différents " maîtres », que ce soit leur langue (le latin, l'italien, le français) ou encore leurs valeurs. (Nous ne reviendrons pas sur cette forme particulière d'attachement à la République qui a amené des milliers de Corses à participer à l'empire colonial français ou encore, dans l'histoire récente de la Corse, comment l'attachement des insulaires à la France s'est manifesté plus particulièrement lors de l'épisode du Serment de Bastia, en 1938 : face à la menace de l'irrédentisme italien qui voulait reprendre la Corse à la France, le président des anciens combattants, M. Ferracci, fit une allocation devant le monument aux morts de la Première Guerre mondiale, plus connue sous le nom de "Serment de Bastia » (1938), dans laquelle il déclara : «Face au monde, de toute notre âme, sur nos gloires, sur nos tombes, sur nos berceaux, nous jurons de vivre et de mourir Français ».)

\footnotetext{
${ }^{64}$ Anne Meistersheim, Figures de l'âle, op. cit., p. 142.

65 Ibid., p.144.
} 
L'île peut alors apparaitre comme une heuristique de la complexité, c'est-à-dire comme un "laboratoire pour la pensée complexe ». Pourquoi ? Parce que formant système, l'île en général nécessite, pour la comprendre, que l'observateur, que le chercheur relie les différents savoirs existant sur elle et identifie les relations qui la lient à son environnement.

\subsection{La construction d'une identité collective corse : la corsitude}

Le sentiment d'identité collective en Corse ne repose pas seulement sur l'appartenance territoriale que nous avons désignée par le terme d'îléité. Elle repose également sur ce qu'on appelle la corsitude. Cette corsitude s'explique par l'existence d'une diaspora importante et dont l'exil tient à de multiples raisons historiques, économiques, individuelles qui se combinent. Ce départ ne signifie pas pour autant une rupture définitive avec la Corse, avec la terre des anciens. Cependant le sentiment d'insularité disparaît alors car l'ex-îlé va vivre d'autres expériences continentales, celles-làa ${ }^{66}$. L'îléité va alors évoluer (mais non pas disparaitre car l'ex-1̂lé se voit souvent revenir, à l'heure de la retraite...) pour prendre la forme de la corsitude. Cependant si le lien avec la terre n'est plus vécu de façon quotidienne, il reste fortement présent dans l'imaginaire des ex-îlés : «depuis toujours, l'homme d'ici a entretenu avec sa terre une relation passionnelle et mystique $\gg{ }^{67}$.

Nous retrouvons ici donc cette boucle récursive entre identité et territoire qui peut être complexifiée encore, si nous considérons la corsitude telle qu'elle est définie par Marie-Catherine MaroselliMatteoli :

Osmose mystérieuse de trois éléments interdépendants : la terre, les hommes et les valeurs, fille de leur douloureuse union et de leur séculaire dialogue, la corsitude est l'expression morale de la mémoire des âges, de la permanence et de la continuité rassurante de notre communauté. Cette influence réciproque, pétrie à tout jamais dans le moule de sa réalité, lui confère son unité distincte, et, aux êtres vivants

6r Enfin, dans la plupart des cas, car il peut aussi arriver que l'exil se fasse vers une autre île.

67 Marie-Catherine Maroselli-Matteoli, La reconquête de la dignité, Ajaccio, La Marge Éditions, 1990 , p. 57. Cet ouvrage est préfacé par le philosophe Jean-Toussaint Desanti. 
qui, s'ils partagent le sort commun à la condition toute entière, la conscience de leur authentique singularité. Ce langage obscur entre les profondeurs de la terre, le sang des hommes et leurs certitudes immuables, a force de lien sacré ${ }^{6.8}$.

La corsitude porte en elle une contradiction en ce sens où elle peut se vivre à la fois dans l'île mais aussi en dehors de l'île, dans une forme déterritorialisée. C'est-à-dire que la corsitude est un sentiment d'identification à la communauté corse qui peut exister même en dehors du territoire corse et insulaire, même si ce n'est pas toujours sereinement. Nous pouvons donc la considérer comme une identité dé-territorialisée, dans une certaine mesure, qui peut exister sans confrontation directe avec le territoire auquel elle se réfère pourtant. Mais cela ne veut pas dire qu'elle serait vidée de sa substance, au contraire. Elle est riche de pratiques culturelles, dont la principale est l'utilisation de la langue corse et de référents spécifiques que nous verrons plus loin.

La corsitude porte en elle la même contradiction que l'îléité : elle est à la fois ouverture et fermeture. Fermeture sur l'univers clos des références à la terre sacrée, au caractère immuable de cette identité telle que l'a évoqué Marie-Catherine Maroselli-Matteoli, mais elle est ouverture sur l'extérieur comme elle le précise aussi : « la corsitude est en soi une richesse, un temps de gloire, une fenêtre ouverte sur toutes les composantes du monde ${ }^{69}$.

Nous avons déjà précisé que nous nous inscrivons ici dans une conception de l'identité corse qui rejette l'essentialisme et le naturalisme pour opter pour une approche constructiviste de la corsitude, de l'identité collective corse. Ceci a donc des conséquences sur notre façon de définir la corsitude. Notre postulat de base sera donc que la corsitude ne peut passer par un droit du sol, pas plus que par un droit du sang ${ }^{70}$. La corsitude tient de l'auto-identification : un individu se définit comme Corse car il adhère à un ensemble de comportements, d'attitudes, de références, à une langue aussi, dans lesquels il se reconnait, et il se réfère

68 Ibid., p. 57.

69) Ibid., p. 63.

70) Si le terme d'ethnie a pu être utilisé notamment par Max Siméoni dans ses interventions au nom de l'ARC (Azzione per a rinascita corsa) dans les années soixante, aujourd'hui, ce terme n'est plus employé ; on lui préfère la notion moins connotée et beaucoup plus ouverte de " peuple ». 
à un territoire particulier, dans des proportions variables et qui lui sont propres $^{71}$.

Iléité et corsitude ne sont pas nées ex nibilo, elles s'inscrivent dans une perspective diachronique. En effet, cette relation particulière à un territoire que nous étudions n'a pu naître que du fait des événements successifs qui ont eu une influence sur les habitants de cette île, des différentes passations et transactions dont elle a fait l'objet au cours des siècles et qui ont nourri un sentiment de résistance et, parfois, de repli sur soi. Cette dimension historique explique à la fois le caractère unique de l'identité collective corse et la diversité de ses univers de référence.

À partir de nos observations, il apparaît que l'identité corse n'est pas forcément quelque chose de très structuré. Chaque acteur y met de sa propre identité personnelle ainsi que les représentations de ce qu'il projette comme étant les caractéristiques de la communauté dans laquelle il se reconnaît et dont il va revendiquer l'appartenance et la pérennité.

L'identité collective s'incarne dans des pratiques culturelles particulières qui s'appuient les unes sur les autres et qui se sont transmises tout au long de l'histoire d'une communauté donnée : l'identité collective corse repose sur une identité culturelle. Là encore, et peut-être surtout, apparaît l'importance de la mémoire dans la transmission des savoirfaire, des savoir-dire, du savoir-être ensemble.

Certains auteurs, comme Béatrice Giblin, récusent l'idée d'une permanence de l'identité culturelle corse.

Affirmer la permanence de l'identité culturelle corse, préservée par l'isolat de l'insularité, n'a guère de fondement quand on veut bien se souvenir de l'importance de l'émigration des Corses tant à l'étranger que sur le continent (trois Corses sur quatre vivent sur le continent), comme si cette émigration n'avait eu aucune influence sur l'évolution de la société insulaire. Il n'existe pas d'identité culturclle immuable, mais affirmer cette permanence a sans doute alimenté la représentation ethnique de la nation corse de certains militants nationalistes, « nous " contre « les autres $»^{72}$.

71 Se pose alors la question des nouveaux arrivants sur le territoire corse. Mais cette question est trop vaste pour que nous puissions l'aborder dans cet article.

72 Béatrice Giblin, "Les nationalismes régionaux en Europe ", Hérodote : revue de géographie et de géographie politique, $\mathrm{n}^{\circ}$ 95, $4^{\mathrm{c}}$ trimestre 1999, p. 17. 
Loin de nous l'idée de considérer que, effectivement, l'identité culturelle corse serait quelque chose d'intangible. Toutefois, il nous semble qu'il est abusif de considérer que cette identité culturelle scrait une construction ethniciste utilisée par les seuls élus nationalistes pour justifier la légitimité de leur action politique. Nous adhérons à l'idée qu'il ne saurait y avoir d'identité figée. Mais cela n'exclut en rien l'existence de points de repères identitaires, de référents dont certains vont perdurer et d'autres disparaittre ou apparaitte. Tous participent de la construction d'une identité culturelle corse qui, quand elle est évoquée ou mobilisée fait appel à un univers de référents communs à tous les insulaires et non insulaires se revendiquant de cette identité culturelle particulière. Notre propos sera donc ici d'en présenter certains qui nous semblent pertinents dans le cadre de notre questionnement, mais sans pour autant prétendre ni à l'exhaustivité ni à l'objectivité absolue. Les points évoqués le sont à partir de notre propre observation du système culturel corse et de la façon dont il nous semble fonctionner aujourd'hui.

L'identité culturelle corse repose sur la mobilisation de ce qu'on peut appeler la culture régionale corse. Ce terme de "culture régionale » fait référence à la région comme territoire de vie. Nous ne chercherons pas ici à donner une définition stricte à ce que peut être une culture régionale, l'exhaustivité des traits qui en seraient caractéristiques ne nous semblant pas possible à atteindre. C'est pourquoi l'approche proposée par Christian Bromberger et Mireille Meyer nous paraît à la fois la plus large et la plus représentative des réalités vécues par les acteurs qui s'engagent pour la préservation et la pérennisation des cultures régionales:

\begin{abstract}
La notion de «culture régionale " [...] connote, dans des proportions variables, un art de vivre ancré dans un territoire, un sentiment de pays, un "entre soi ", un folklore pittoresque volontiers exhibé à des fins touristiques, la défense et la promotion d'une langue et de ses expressions singulières, parfois une revendication d'autonomie, voire, récemment, d'indépendance ${ }^{73}$.
\end{abstract}

L'identité culturelle repose principalement sur la pratique de la langue corse mais intègre également d'autres éléments qui sont plus diffus dans la communauté insulaire.

Christian Bromberger et Mircille Meyer, « Cultures Régionales en débat », Ethnologie française, «Cultures régionales. Singularités et revendications », vol. XXXIII, $\mathrm{n}^{\circ} 3$, 2003 , p. 357. 


\subsubsection{La langue, fondement de l'identité culturelle corse}

Dans les phénomènes et pratiques identitaires, sans vouloir hiérarchiser à tout prix, il nous semble cependant que la langue est de toute première importance : «l'emblème majeur de la singularité et des revendications régionales est [...] la langue $»^{74}$. Elle permet de dire « je suis »: le " quoi " dans « je suis quoi » est nourri par la culture et les représentations sociales nourries et transmises collectivement. Elle est également le vecteur de la transmission d'une histoire commune qui fonde l'identité, comme nous l'avons vu plus haut, et sa pérennisation. Pour Christian Bromberger et Mireille Meyer, l'utilisation de la langue locale a une fonction sociale particulière : ce n'est pas tant de promouvoir la langue en elle-même pour en faire un moyen de communication entre différentes personnes, mais c'est bien un outil de revendication, qui permet « d'exprimer une adhésion affective ou un souci patrimonial ${ }^{75}$. En effet, si la langue disparait, la culture disparaît. Elle est l'élément essentiel par lequel se transmet l'ensemble des traits culturels corses, par lequel se définit aussi en partie l'identité collective ; mais elle est aussi ce par quoi la culture et l'identité évoluent. Si sa pratique diminue, l'identité pourra peut-être se perpétuer, mais elle sera amoindrie, car elle perdra une partie de son fondement culturel, le lien avec son histoire lointaine et son patrimoine. Mais on peut se reconnaître dans l'identité corse sans pour autant être corsophone.

La langue corse est ce qui permet de se dire corse, elle participe de l'auto-définition - sò corsu ${ }^{76}$ - et possède une fonction fondamentale dans la construction de l'imaginaire collectif. Mais elle ne suffit pas pour faire une identité culturelle, elle est secondée en cela par d'autres éléments qui, avec la langue, ont contribué et contribuent à construire au jour le jour l'identité collective corse.

\subsubsection{Les autres dimensions de l'identité culturelle corse}

Le patrimoine est l'un des éléments fondamentaux de l'identité culturelle, car il permet à la mémoire collective de s'exprimer et, récursivement, de se

\footnotetext{
$74 \quad$ Ibid., p. 358.

75 Ibid., p. 359.

76 «Je suis corse. »
} 
nourrir de ce qui lui est donné à voir quotidiennement. Les vestiges plus ou moins anciens sont les traces les plus tangibles, à la fois de l'histoire et de la vie quotidienne d'une communauté depuis le peuplement d'un territoire, et ceci à travers les âges. L'exemple le plus parlant est bien sûr celui des statues de Filitosa, en Corse-du-Sud. Lady Dorothy Carrington, célèbre anthropologue spécialiste de la Corse, a débarqué sur l'île (qu'elle ne devait quasiment plus quitter) en 1948 pour voir ces statues qui datent de la moitié du $\mathrm{II}^{\mathrm{e}}$ millénaire avant notre ère ${ }^{77}$.

La transmission du patrimoine est également essentielle pour nourrir le sentiment de communauté de destin qui est partie intégrante du sentiment identitaire. Mais il ne faut pas prendre en considération seulement le patrimoine des monuments historiques : le patrimoine quotidien, les simples demeures familiales sont autant de témoignages. D'où l'émotion ressentie dans les villages désertés à la vue des maisons centenaires délabrées, dont les héritiers nombreux et dispersés ne connaissent pas l'existence ou n'ont pas pu s'entendre sur la prise en charge des réparations nécessaires à la survie de ces bâtisses. Mais, pour autant, le village reste dans l'imaginaire collectif corse comme une référence prégnante, et ce, malgré la forte urbanisation qu'a connue la Corse ces dernières décennies.

Pour les Corses urbanisés, [persiste] le maintien de liens réels et symboliques encore forts avec les villages d'origine: c'est vers eux que l'on se rend, dès qu'un week-end prolongé ou les vacances et congés le permettent, même si, pour les adolescents, la tentation est forte de revenir à la ville où ils trouvent les équipements de sportif et de loisirs auxquels ils sont attachés ${ }^{78}$.

Il existe un patrimoine spirituel et un patrimoine social liés à la vie quotidienne. Certains cultes sont importants. Ils représentent un patrimoine spirituel qui se traduit aussi sur le plan architectural. Le culte

77 Dorothy Carrington, La Corse, Paris, Arthaud, 1999. Sur la découverte des statues, voir les pages 37 à 54 .

78 Georges Ravis-Giordani, «La Corse : culture régionale ? Culture régionalisée ? ", Ethnologie Française, "Cultures régionales. Singularités et revendications», vol. XXXIII, $\mathrm{n}^{\prime 2} 3,2003$, p. 455. Il est d'ailleurs intéressant de noter que les individus ou les familles qui s'installent en Corse et désirent y demeurer (car ils s'identifient à cette région et veulent être identifiés comme Corses) recherchent l'ancrage villageois, en acquérant ou en essayant d'acquérir une maison au village. 
de la mort par exemple, en Corse. La mort tient une grande place dans la société insulaire ; en témoignent la force des rites mortuaires (notamment la tradition des voceri...) et les monuments funéraires qui sont tellement intégrés dans la vie quotidienne, dans le paysage quotidien. Ici, les tombeaux familiaux, parfois somptueux, sont intégrés à l'architecture globale des villages, ou bien fleurissent au milieu des montagnes, sur les terres de la famille. Pour Marie-Jean Vinciguerra ${ }^{79}$, ce culte de la mort va de pair avec une culture de la tragédie ${ }^{80}$ :

[La Corse] est marquée par les tragédies, elle est marquée parce que la vie était difficile, parce qu'il y a eu les hécatombes de la guerre de 14. [...] Quand ma mère voulait rire, sa mère lui disait : " ton frère est revenu? ». Il avait été tué à la guerre. Toute une vie comme ça, bon. Et la convivialité, elle était aux enterrements, tout le monde va pour enterrer les morts. Dans mon village de Ghisoni, on entre dans les dieux lares. C'est toutes les vieilles avec leurs foulards. Ce n'est pas une culture de la joie, ce n'est pas, non! On le sait d'ailleurs, il y a les voceri, les lamenti, les chants de la vengeance.

Dans ces expressions d'un imaginaire collectif insulaire, fortement imprégné de douleur et de mysticisme, l'image de la femme joue un rôle important. Dans cette société patriarcale, la femme, il faut le reconnaitre, était en retrait, sauf quand elle accédait au statut de mère : dès lors elle possédait un pouvoir certain sur le cercle familial. D'ailleurs, dans la tradition vendettaire, c'est elle bien souvent qui appelait à la réparation du crime commis contre l'époux ou le fils. Certaines femmes ont occupé une place importante dans l'Histoire corse (Vannina d'Ornano, Danielle Casanova, etc.) ainsi que dans l'imaginaire collectif insulaire (soit en tant que personnage comme Colomba ou en tant qu'écrivaine comme Marie

79 Élu du groupe majoritaire de droite Le Rassemblement à l'Assemblée Territoriale de Corse.

80 Au cours de l'un de mes déplacements pour ce travail de recherche, j’ai eu l'occasion d'assister à une représentation de l'Antigone d'Anouilh. Cette pièce avait été traduite en grande partie en corse, quelques passages étant conservés en français, afin que les spectateurs non corsophones et non connaisseurs de la pièce puissent suivre l'histoire. Il m'est alors clairement apparu que les passages en corse étaient de loin ceux les plus marquants non pas parce que seuls les passages les plus poignants avaient été traduits mais bien parce que leur diction en langue corse portait en soi le souffle de la tragédie. On sentait les spectateurs plus enclins à en partager le poids lors des passages en langue corse. 
Susini). Mais au-delà de la place de la femme dans la société corse, c'est le ressort symbolique qu'elle constitue qu'il est intéressant de considérer. En effet, dans le discours, le territoire de la Corse est souvent présenté avec des connotations féminines et maternelles. Pour Jackie Poggioli, enseignante et journaliste :

\begin{abstract}
Quand on représente la Corse, symboliquement, l'image la plus immédiate est celle très maternelle d'une femme en noir, avec le mandile ${ }^{81}$. Il est évident que l'espace insulaire est fortement féminisé, mais ce n'est pas spécifique à la Corse. Dans l'imaginaire universel, l'île et la femme ont partie liées [sic], et dans les cosmogonies des cultures insulaires, les légendes associent toujours la féminité et l'île ${ }^{82}$.
\end{abstract}

Pour elle, cette analogie du territoire de l'île et du corps féminin est plus présente chez les auteurs insulaires exilés pour qui cet exil influence la façon de se représenter la Corse.

Pour eux, elle est, avant la terre de l'enfance, et de ce fait, elle est associée étroitement à la figure maternelle, aussi prégnante pour les hommes que pour les femmes, même si l'apport de ces dernières est différent. Dans cette littérature d'exil, la terre-mère est un espace très ambivalent, à la fois protecteur et mortifère vers lequel le retour s'apparente à une régression foetale, et auquel il faut savoir s'arracher pour vivre ${ }^{83}$.

Aujourd'hui l'expression culturelle, notamment musicale mais pas seulement, est aussi un moyen de faire revivre des éléments marquants du passé, de les pérenniser dans la mémoire collective. Ainsi Jean-Valère Geronimi $^{84}$ évoque-t-il cet événement tragique qui est encore dans la mémoire des gens de Calacuccia et transmis par la tradition orale :

Je suis de la moitié du siècle, né dans un petit village qui s'appelle Calacuccia, où il y avait une identité très forte et une mémoire très forte, transmise par l'oralité, sur une époque de notre histoire où les Français nous avaient conquis et avaient pendu des gens et déporté des gens. Donc on parlait de ces fameux onze pendus. Donc c'était une transmission orale qui était très forte.

81 Mandile : foulard.

82 Jackie Poggioli, « De la féminité de l'identité corse ", dans Ange Casta (dir.), La parabole corse, op. cit., p. 101.

83 Ibid., p. 101.

${ }^{84}$ Élu du groupe Corsica Nazione à l'Assemblée Territoriale de Corse. 


\section{NPSS, VOLUME 2, NUMÉRO 1, 2006}

Le groupe polyphonique A Filetta s'est fait l'écho de la transmission de cet événement dans une pagbjella (chant polyphonique) intitulée $A$ Pagbjella di l'Impicati (La paghjella des pendus) ${ }^{85}$. Ceci n'est pas exceptionnel car tout un pan de la culture corse s'exprime de façon orale, à travers les chants et les contes. La musique sacrée a une importance certaine; rappelons que l'hymne corse est un chant liturgique : Dio vi Salve Regina.

Toute identité collective (notamment, pour ce qui nous intéresse plus particulièrement, territoriale, nationale, régionale, locale, politique) est véhiculée et se pérennise par la culture. Mais celle-ci ne doit pas être envisagée de façon élitiste, car elle est aussi quotidienne (à travers les savoir-faire) et populaire (les chants, les danses, les veillées, les différents dialectes locaux, les proverbes, les expressions). L'expression populaire de l'identité culturelle est sans doute pour une grande partie ce qui fait sa vivacité, sa vitalité, son principe de vie et ce qui permet sa plus grande pérennisation (même si on est conscient, notamment pour tout ce qui a trait à la langue, que la faiblesse des locuteurs est une véritable épée de Damoclès quant à la survivance des langues régionales, cela est valable tant pour le corse que pour le breton, l'occitan, etc.). Et ce qui fait qu'elle se pérennise est le fait qu'elle admette, autorise et pratique l'intégration de facteurs exogènes (personnes, ambiances, musiques...), c'est-à-dire tout ce qui fait le processus de (re)construction identitaire.

Cette reconstruction identitaire est d'ailleurs défendue par des élus qui ne sont ni proches des nationalistes, ni des autonomistes mais qui revendiquent la nécessité de la défense de l'identité corse, dans une attitude qui ne soit pas passéiste mais qui intègre au contraire la nouveauté.

Les questions culturelles ont fait et font toujours partie des problèmes fondamentaux de la Corse tant sur des questions de promotion d'une culture particulière qui n'a pas toujours été reconnue en tant que telle (prenons, par exemple, la polémique sur l'existence d'une littérature corse, laquelle est aujourd'hui peu à peu reconnue) mais également sur les infrastructures : comment dans une société enclavée et aux infrastructures peu développées faire en sorte que la culture soit accessible à tous?

85 Paroles : Ghjuvan-Teramu Rocchi, Musique : Ghjuvan-Claudiu Acquaviva, dans A Filetta, Intantu, Virgin, 2002. 
À partir des représentations les plus communes qu'ont les Corses que nous avons interrogés sur leur propre identité, il nous a semblé intéressant d'utiliser une notion qui rende compte de ce qui fait à notre avis la singularité du sentiment identitaire corse : la corsitude. En effet, à partir de nos entretiens, de nos lectures et de nos observations sur le terrain, s'est forgée l'idée que la corsitude ne pouvait se concevoir comme un sentiment uniforme et qu'une vision trop homogène pouvait au contraire vider cette identité de sa substance.

Ainsi, il nous semble qu'on peut observer ce que nous appellerons une endo-corsitude et une exo-corsitude - que nous distinguons ici pour une meilleure compréhension et pour mieux incarner les deux aspects de la corsitude que nous avons déjà évoqués plus haut - lesquelles sont en interaction.

L'endo-corsitude est le fait de ceux qui vivent sur l'île. L'exocorsitude est celle des exilés (ex-îlés), de ce que nous pouvons appeler la " diaspora » de par la dispersion géographique des Corses de l'extérieur, mais dont l'implication et l'intérêt pour le devenir de l'île restent vifs. L'endo- nourrit l'exo- en lui transmettant la langue et tout ce qui est information identitaire et culturelle qui fait que l'exo-corsitude peut continuer à alimenter la représentation qu'elle a d'elle-même, de son moi collectif. Réciproquement, l'île peut trouver quelque intérêt à ce que les exilés peuvent lui apporter : rôle d'ambassadeur, par exemple, ou rôle de propagation et de reconnaissance par les autres de certains référents. Pour Jean-Toussaint Desanti, «[n]ous sommes toujours dans ce rapport à la fois d'exclusion et d'intériorité. L'intérieur et l'extérieur se tiennent. La notion de frontière doit être pensée entièrement, elle n'est pas une ligne de séparation mais une relation mobile ${ }^{86} . \mathrm{La}$ pérennisation de la transmission de l'identité corse par la diaspora est un élément non négligeable. En effet, il y a plus de Corses (se définissant comme tels dans la vie de tous les jours) vivant en dehors de l'île que de Corses insulaires. L'affirmation de leur identité s'illustre par la création d'amicales, d'associations, mais aussi, dans le cercle plus restreint de la famille, à travers la transmission de certains référents culturels.

Par ailleurs, si la notion d'identité n'a pas toujours fait partie du registre lexical de l'action politique et publique en Corse, il nous semble

86 Jean-Toussaint Desanti, «Les lieux sont tombés sur la tête... », op. cit., p. 11. 


\section{NPSS, VOLUME 2, NUMÉRO 1, 2006}

qu'un certain nombre d'éléments corroborent l'hypothèse que le fait identitaire s'est peu à peu introduit dans la sphère publique et politique en Corse pour arriver aujourd'hui à une situation où parler d'identité fait partie du langage commun de la politique sur l'île.

La revendication identitaire est apparue sur le plan discursif comme la clé de voûte de tous les mouvements et phénomènes sociaux d'importance en Corse depuis la fin de la Seconde Guerre mondiale, en partant de la revendication du mouvement du 29 novembre 1959, qui protestait contre la volonté du gouvernement de l'époque de supprimer les chemins de fer locaux, en passant par les revendications à connotation écologique des années 1960-1970 (Argentella, Boues rouges) pour arriver aujourd'hui aux revendications de nature économique (défiscalisation, économie identitaire, agriculture identitaire, prime d'insularité...). Pour ce qui est du domaine politique, la question s'est posée de manière très abrupte en Corse, puisque la revendication nationaliste se nourrit de l'idée qu'il existe un peuple et une nation corses fondés sur une histoire, une identité et une volonté de vivre ensemble sur le sol corse. Aussi la défense de l'identité corse s'est trouvée être au centre du combat politique, entre les nationalistes et les différents gouvernements qui se sont succédés.

Plus près de nous, si le processus de Matignon a pu être un enjeu de pouvoir au niveau national, au niveau local, nous avons pu constater l'existence d'un certain consensus (revendiqué au moins) autour de la volonté de préserver l'identité culturelle corse et de la nécessité de prendre en main le développement de la Corse qui a entrainé deux choses : d'une part, la rupture, ou du moins le décalage entre les leaders politiques locaux et leur direction politique nationale; d'autre part, une démarche consensuelle des élus, certains mettant de côté quelques-unes de leurs convictions les plus fortes afin que le processus, la démarche aillent de l'avant, la concession ayant souvent été faite sur la question de la langue obligatoire (certains préférant qu'elle soit facultative mais ayant quand même voté pour obligatoire). Aussi la défense de l'identité corse est apparue comme n'étant plus le domaine réservé des nationalistes corses, pour autant qu'elle ne l'ait jamais été.

En effet, certains élus que nous avons rencontrés, non nationalistes, ont même des propos très durs face à l'idée que les nationalistes pourraient être les seuls détenteurs de l'argumentaire identitaire en 
Corse, jusqu'à leur faire le procès d'une instrumentalisation commerciale de l'identité corse.

L'identité est le commerce des nationalistes, elle n'a jamais été la chose des nationalistes. Je suis depuis tout le temps aussi identitaire que n'importe quel nationaliste. Mais je n'en ai jamais fait commerce. C'est tout à fait différent. [...] Et nous sommes aussi identitaires que n'importe quel nationaliste. Ça, on n'a pas besoin des nationalistes pour être Corse et pour parler le corse aussi bien qu'eux, si ce n'est mieux ${ }^{87}$.

D'autres sont plus nuancés, considérant qu'«il n'y a pas que les nationalistes qui défendent l'identité », comme le conseiller territorial socialiste Jean Motroni.

Le leader nationaliste Jean-Guy Talamoni, quant à lui, lie la transversalité de l'identité corse et la mobilisation des élus territoriaux dans le processus de Matignon :
Je pense que l'identité corse, elle n'est pas réservée aux nationalistes. L'identité corse a influencé aussi les choix de tous les autres élus, qui ne sont pas nationalistes, tout à fait, bien sûr. Je crois que ce qui a dû motiver les élus de la Corse en général, c'est bien entendu à un moment donné l'intérêt de la Corse qui a connu des décennies de conflits, qui ont suivi des décennies d'ailleurs de catastrophes économiques, sociales et culturelles.

L'identité revendiquée ici est présentée comme unifiée, voire unificatrice, pour faire face à l'Autre. Or, il n'existe pas une identité corse uniforme et univoque, mais bien des corsitudes, qui s'appuient sur des expériences différentes de ce sentiment d'appartenance à la communauté corse, dans sa forme insulaire ou dans sa forme ex-îlée. Mais, pour autant, ces diversités ne sont pas incompatibles avec une vision globale de l'identité collective corse, le plus souvent mobilisée avec force quand il s'agit de faire face aux autres (communautés, peuples, nations). Le fait que cette identité collective soit protéiforme s'explique également par la façon dont les Corses se représentent leur propre territoire - divers et compartimenté - en raison de sa géographie montagneuse.

87 Joseph Antona, Maire de Quenza, Conseiller Territorial, groupe Le Rassemblement. 


\section{L'identité collective nourrit la représentation du territoire : " une montagne dans la mer »}

Si la Corse est bien une île de la Méditerranée, elle y occupe une place à part. Plus que les Baléares, Malte et la Sicile, et même que sa plus proche parente, la Sardaigne, elle est, selon la formule attribuée à Friedriech Ratzel, « une montagne dans la mer » ${ }^{88}$. La chaine montagneuse, qui sert à la fois de frontière naturelle et administrative entre la HauteCorse et la Corse-du-Sud, marque une séparation qui a eu des conséquences sur l'histoire et le développement des deux parties de l'île.

\subsection{De la multidimensionnalité de l'identité corse}

Nous avons vu plus haut que l'îléité ne pouvait se concevoir sans une nécessaire relation à l'Autre qui permet au système insulaire de se définir et de se construire. L'analyse systémique nous permet de comprendre qu'un système, quel qu'il soit, est rarement fermé, son ouverture est la condition sine qua non de sa pérennisation. Appliqué au système identitaire corse, cela signifie qu'il ne peut se définir ex nibilo, mais qu'il s'est élaboré au cours de l'histoire en intégrant différents apports qui lui ont permis de se définir comme un système identitaire, culturel à part entière.

S'il est difficile d'établir une hiérarchie des références constitutives de l'identité collective corse, il nous semble cependant que l'identité corse, dans une première approche, se vit dans sa méditerranéité, définie comme "l'ensemble des caractéristiques communes, plus ou moins rémanentes que le mare nostrum a imposées aux civilisations qui la bordent ${ }^{89}$. Cette méditerranéité historique de la Corse trouve ses origines dans l'arrivée des Phocéens et la fondation d'Alalié (qui deviendra Alalia avec les Romains puis Aléria) au milieu du $\mathrm{VI}^{\mathrm{e}}$ siècle avant J.-C. Cet héritage grec se retrouve aujourd'hui dans nombre de similitudes partagées entre la Corse et la Grèce ${ }^{90}$.

Comme le rappelle Georges Ravis-Giordani, «l'impossible Mezzogiono», (Méditerranéennes, Éditions Albiana, Association Méditerranéennes, $\mathrm{n}^{\circ} 12$, été 2001, p. 28). Il précise dans la note se reportant à cette référence : "Dans son essai, "La Corse, étude anthropologique", publié dans les Annales de Géographie en 1899, Ratzel parle "d'un pays de montagnes dans la mer", ce qui n'est pas la même chose ».

89 Jean-Louis Andreani, Pour comprendre la Corse, op. cit., p. 20-21.

90 Pour plus de détails sur ces similitudes, voir l'article très intéressant de Pascal Maccioni, «La Corse : la plus grecque des terres françaises », Confluences Méditerranée, $\mathrm{n}^{\circ} 4$, automne 1992. 
Plus globalement et en regardant la Corse dans sa modernité,

[1]a Corse n'est pas [...] plus proche du Sud de la Méditerranée que du Nord. Elle est certes une zone de transition entre "Nord " et "Sud", où les caractères sociologiques d'une population méditerranéenne permettent un rapprochement incontestable avec les autres rivages méditerranéens. Mais il ne faut pas trop s'engager dans une voie qui trahirait la réalité d'une société corse contemporaine, totalement intégrée à l'Europe de l'Ouest, celles des consommateurs ${ }^{91}$.

Cet aspect anthropo-historique ne peut masquer la réalité géographique et géologique : la Corse occupe une position centrale dans la Méditerranée occidentale, ce qui la lie à la péninsule italienne, et notamment à l'archipel toscan, puisque les fonds marins sont peu profonds entre le Cap Corse et le plateau toscan alors qu'ils font 2500 mètres de profondeur quand on se tourne vers le continent français. Géographiquement, historiquement, culturellement, l'identité corse balance entre l'Italie et la France, intégrant les apports de chacune.

2.1.1. L'identité « historique » corse $:$ le dialogue entre l'Italie et la France

L'identité corse englobe une part d'italianité qu'on peut qualifier, avec Joseph Martinetti, d'《 historique $»^{92}$, car les épisodes pisans et génois ne sont pas sans avoir laissé de traces dans la société et la culture corse ${ }^{93}$, notamment en matière linguistique. Certains aspects religieux ne sont pas absents non plus, si on se réfère aux rituels processionnaires, comme la granitola ${ }^{94}$, à la dévotion particulière à certains saints et à l'importance que revêtent encore certaines fêtes particulières comme Pâques ou l'Assomption. Mais cette italianité n'est pas uniforme : la Corse est culturellement et historiquement proche de la Toscane mais

91 Joseph Martinetti, Insularité et marginalité..., op. cit., p. 41.

92 Ibid., p. 42.

93 Nous ne développerons pas ici les aspects strictement historiques des relations entre les royaumes proto-italiens et la Corse, car plus que les événements en eux-mêmes, ce sont leurs conséquences qui nous importent ici. De plus, de nombreux ouvrages ont été écrits sur ces épisodes. Voir, entre autres, Paul Arrighi et Francis Pomponi, Histoire de la Corse, Paris, PUF, Que sais-je ?, 1993 ; Roger Caratini, Histoire du peuple corse, Paris, Criterion, Histoire et histoires, 1995 ; Joseph Martinetti, Insularité et marginalité...., op. cit. ; Janine Renucci, La Corse, Paris, PUF, Que sais-je ?, 2001 ; Michel Verge-Franceschi, Histoire de la Corse, le pays de la grandeur, Préface d'Emmanuel LeroyLadurie, Paris, Éditions du Félin, 1996. La liste n'est, bien entendu, pas exhaustive.

${ }^{94}$ Danse processionnaire au tracé spiralé. 
aussi et surtout de la Sardaigne, que l'on présente souvent comme sa sœur ou sa cousine. Toutes deux ont des référents communs tant sur le plan identitaire que culturel ou même historique. Pour Joseph Martinetti,

$$
\begin{aligned}
& \text { la proximité entre la Corse et la Toscane, et la Corse et la Sardaigne est } \\
& \text { à la fois objective (réelle) et subjective (vécue). Ses liens culturels, } \\
& \text { héritage d'une histoire commune, sont aujourd'hui encore trop forts } \\
& \text { pour être dissimulés derrière les paravents d'une Corse autocentrée } \\
& \text { depuis l'aube des temps, ou d'une corse francisée comme un Nouveau } \\
& \text { Monde }^{95} \text {. }
\end{aligned}
$$

Même si aujourd'hui persistent ces référents italiens dans l'identité collective, ils ont cédé une large place à d'autres, que l'on peut désigner à travers le terme de "francité " ${ }^{96}$. Cette " part française de l'identité corse $^{97} \gg$ tient au rattachement de l'île à la France au XVIII ${ }^{\mathrm{e}}$ siècle, ce qui a eu des conséquences en matière économique, institutionnelle, sociale, culturelle, de styles de vie, etc., implications qui ne doivent être négligées si nous ne voulons pas amputer l'identité et la culture insulaires. Le roman de Paul-Michel Villa, La maison des Viale ${ }^{98}$, illustre bien le passage d'une Corse fortement ancrée dans la culture italienne à une Corse qui s'ouvre à l'univers français, à travers le parcours de la famille Viale, qui nous montre comment les élites se sont tournées vers la France à partir de la seconde moitié du XIX ${ }^{\mathrm{e}}$ siècle, alors que, administrativement, la Corse appartenait déjà à la France depuis 1768.

D'ailleurs ce sentiment d'appartenance française ou de double appartenance corso-française est toujours bien vivant si nous nous référons à un sondage réalisé par Louis Harris et publié par le magazine Corsica de janvier 2000 et selon lequel $53 \%$ des personnes interrogées se disent autant corses que françaises, $15 \%$ plus corses que françaises, $14 \%$ seulement françaises, $8 \%$ plus françaises que corses, $8 \%$ seulement corses, $2 \%$ ne se prononçant pas, soit au total $90 \%$ qui se reconnaissent une identité française aussi réduite fût-elle, tout en étant

95 Joseph Martinette, Insularité et marginalité..., op. cit., p. 44.

${ }^{96}$ Ce mot, bien que connoté idéologiquement dans certains contextes, est ici à considérer comme une catégorie heuristique, construite selon le même schéma que méditerranéité ou italianité, sans autre raison que la compréhension du phénomène identitaire.

${ }^{97}$ Jean-Louis Andreani, Pour comprendre la Corse, op. cit., p. 22.

98 Paul-Michel Villa, La maison des Viale, Ajaccio, Édition Alain Piazzola, 1994. 
$84 \%$ à avoir conscience de leur spécificité. Mais, aujourd'hui, nous pouvons affirmer que l'identité corse se construit également dans un dialogue avec l'Europe.

\subsubsection{De la modernité de l'identité corse : le dialogue entre Méditerranée et Europe}

Si la Méditerranée peut apparaittre comme la sphère d'appartenance historique, géographique et culturelle de la Corse, s'imposant quasi naturellement, la référence à l'identité méditerranéenne a pu décliner avec le temps. Mais, à travers la mise en place de partenariats économiques, politiques, culturels ou scientifiques, un nouvel élan méditerranéen se fait jour et qui appelle à une modernité de la part méditerranéenne de l'identité corse. Nous sommes là face à une véritable redéfinition de la part méditerranéenne dans l'identité collective corse. L'appartenance commune à cette aire est devenue une source de créativité, notamment culturelle, pour l'ensemble des partenaires des réseaux méditerranéens qui se sont mis en place et que nous avons évoqué chemin faisant.

Cette envie de rencontre et d'action en commun peut trouver son explication dans la reconnaissance de la complexité de la situation de chacun des partenaires (notamment, si l'on s'intéresse plus particulièrement aux territoires insulaires) qui est en résonance et en interaction avec la complexité de la Méditerranée qui est « un ensemble complexe, à la fois Un et Multiple, où règnent des tensions, des contradictions, des affrontements, mais où il demeure néanmoins un sentiment d'appartenance, une part irréductible qui donne vie à un être au monde méditerranéen ${ }^{99}$.

Cet « être au monde » particulier repose sur la relation à l'Autre, perçue comme nécessaire et comme une forme de résistance à un occidentalisme, parfois vécu comme trop individualiste, alors que culturellement la Méditerranée s'est construite sur une socialité très développée, à travers notamment la prédominance des relations de parenté et d'affiliation.

Cette résistance repose sur la créativité de la Méditerranée, nécessaire à la mise en œuvre de cet « être au monde ». Si cette créativité s'exprime de façon très importante en matière culturelle (à travers divers festivals et d'autres types de rencontres, en Corse ou ailleurs dans le bassin méditerranéen), nous pouvons également considérer qu'elle peut

99 Thierry Fabre (dir.), La Méditerranée créatrice, La Tour d'Aigues, Éditions de l'Aube, 1994, p. 9. 
s'exprimer de façon plus politique à travers le rapprochements des élus et la mise en place de projets communs. IMEDOC en est un exemple fort.

Si le volontariste des institutions et des acteurs de terrain (élus, membres du système économique ou culturel) est plus que nécessaire à la mise en place d'une coopération active et efficace, il ne peut se passer d'un vouloir être ensemble. "Ce qui importe en fait, c'est le sentiment d'appartenance à un même ensemble, donc l'existence d'une volonté de se soumettre à un système de règles collectives et de fonder un avenir commun $»^{100}$.

Ces processus de coopération entraînent à notre sens un renforcement de l'identité corse vue comme un dialogue entre Europe et Méditerranée (sans que cela suppose une remise en cause de l'appartenance à l'ensemble national français, car c'est par elle que la Corse fait partie intégrante de l'Union Européenne). Nous pouvons alors voir ce double ancrage institutionnalisé comme une identité-projet : c'est-à-dire que la coopération institutionnelle de la Corse avec d'autres régions européennes et méditerranéennes l'amène à réfléchir sur ce qu'elle est aujourd'hui pour agir dans un avenir proche. C'est parce qu'ils se reconnaissent une double appartenance que les élus vont élaborer des projets d'action dans lesquels la Corse va être partie intégrante et qui vont donc renforcer cette double inscription identitaire.

Le lien entre Méditerranée et Europe peut être source d'un véritable questionnement politique. Ainsi existe-il une réflexion sur une possible méditerranisation ${ }^{101}$ de l'Europe, menée, entre autres, au sein du réseau de Recherches Dynmed. Il s'agirait, à partir de la culture commune de la Méditerranée ( le goût [...] pour une certaine qualité des relations humaines, les valeurs [partagées] ${ }^{102}$ ), de proposer un modèle alternatif de développement économique, rejetant le modèle libéral anglo-saxon et américain et de « construire pour le futur un modèle d'organisation politique qui libère en même temps la créativité et l'initiative, qui

1019) Michel Rombaldi, "La coopération interrégionale en Méditerranée ", dans Anne Meistersheim (dir.), L'ille laboratoire, op. cit., p. 109.

101 Par allusion à la contribution d'Edgar Morin « Penser la Méditerranée et méditerranéiser la pensée ", dans La Méditerranée, modernité plurielle, Paris, Éditions Publisud, 2000.

102 François de Casabianca, "Méditerraniser l'Europe », Corsica Magaz̧ine, août 2001, p. 66. L'auteur de cet article est l'ancien coordonnateur du réseau de recherches Dynmed. 
permette de trouver l'alliage entre culture et économie, de recomposer un humanisme fraternel capable d'éclairer et de dynamiser nos vies $"{ }^{103}$.

De tout ceci nait une interrogation légitime quant à la relation au territoire qui amène à ne pas prendre comme référent unique le territoire propre (l'île) mais à prendre aussi d'autres territoires plus ou moins proches ou lointains, tant sur le plan géographique que culturel, etc. Le processus identitaire, c'est-à-dire la mise en place et la construction de l'identité, sa définition, se crée dans une relation avec des territoires qui sont eux-mêmes en contact, en interactions et en interrelations. Les niveaux sont enchevêtrés et inextricables, car nous ne pouvons pas établir une hiérarchie des interrelations, nous ne pouvons pas nous aventurer à dire laquelle est première par rapport aux autres.

Nous ne pouvons d'ailleurs qu'ajouter des niveaux de complexité puisque, à l'intérieur du territoire insulaire, force est de constater la diversité des aires de référence : le village, les pieve, la vallée dans lesquels la famille (au sens large) prend sa source. La référence est ici génétique et mythique, car parfois il n'est plus guère de membres de la famille qui " montent » au village (faute d'individus présents sur l'île ou tout simplement suite aux décès successifs) pour entretenir la maison de famille, mausolée symbolique, perdu dans une nébuleuse généalogique. C'est pourquoi nous ne pouvons douter que l'identité est intrinsèquement liée à la mémoire individuelle et collective. On se définit aussi par rapport à des événements, des personnages appartenant au passé, plus ou moins importants, mais qui sont ancrés dans une histoire commune, à tel point qu'ils transcendent leur simple valeur de repère diachronique. Cette mémoire collective va nourrir les représentations à partir desquelles la communauté étudiée va s'auto-définir. Mais, en Corse, on se définit tout aussi fortement par son appartenance territoriale : le village et la vallée, qui représentent autant d'îles dans l'île.

2.2. L'île est un territoire compartimenté qui se vit dans la diversité : l'île-archipel

La nature montagneuse de l'île, que nous avons déjà évoquée, a des conséquences importantes sur l'organisation sociale à l'intérieur de l'île.

${ }^{103}$ Ibid., p. 66. On retrouve ici l'argumentation développée par Edgar Morin dans plusieurs de ses ouvrages sur la nécessité de penser autrement notre rapport à l'humanité et à l'économie. 


\subsubsection{Vallées et pieve}

Il n'y a pas un territoire corse mais une multitude de terrains de références dont les intérêts seront parfois complémentaires parfois antagonistes. Ces différents territoires se calquent sur les vallées isolées qui ont une importance historique fondamentale et nous amènent à considérer qu'il n'y a pas une identité uniforme du nord au sud de l'île. Ces vallées permettent d'envisager l'identité corse comme un camaieu, un dégradé de couleurs d'une même gamme, ni tout à fait différentes, ni tout à fait les mêmes.

La diversité des expériences de vie en Corse s'explique par la nature montagneuse de cette île. Elle est divisée en vallées qui, isolées les unes des autres, ont favorisé l'émergence d'îles à l'intérieur de l'île, c'est-à-dire qu'elles ont engendré une vision spécifique de leur territoire insulaire que les îliens désignent par "l'île-archipel » : « La Corse n'est pas une île : c'est un archipel dont les détroits, les bras de mer, les passes et les récifs sont des montagnes et des vallées, dont les îles sont les pieve et les îlots des hameaux $»^{104}$.

$\mathrm{Si}$ les pieve sont une réalité historique aujourd'hui disparue, ils demeurent des micro-territoires, appelés micro-régions, dont les spécificités sont défendues par les élus qui en ont la charge. Pour Pierre Chaubon ${ }^{105}$, la spécificité du territoire cap-corsin est réelle par rapport au reste de l'île :

Je suis élu de longue date [...] du Cap Corse. On a appelé ça souvent l'île dans l'île. Surtout que le Cap Corse est une micro-région, aujourd'hui on dit un territoire particulier, certainement puisque les Cap-corsins ont été des marins, les Cap-corsins sont partis plus que d'autres à la conquête ou à la découverte du monde. Et donc, quand ils sont revenus, forcément ils étaient imprégnés d'influences diverses. Donc, le Cap corse en soi, c'est un territoire spécifique. En même temps, il a une conscience aiguë de son appartenance à quelque chose de plus vaste qui est la Corse. Je suppose que ce qui est vrai pour le Cap Corse, peut-être dans des proportions encore plus grandes, parce que c'est le bout, une extrémité, c'est une micro-région particulièrement bien identifiée, mais je suppose malgré tout que ce qui est vrai pour ce territoire-là est vrai pour d'autres.

104 Roger Caratini, La Corse, un peuple, une bistoire, Paris, Éditions de l'Archipel, 2003, (édition revue et augmentée de l'H Histoire du peuple corse, Paris, Éditions Criterion, 1995), p. 9. À travers cet ouvrage, l'objectif de l'auteur est de montrer que les populations de ces territoires isolés ont, au cours de l'histoire, pu se rencontrer, se reconnaitre une communauté de destin sur cette île jusqu’à devenir un peuple.

105 Élu du groupe Corse Social-Démocrate à l'Assemblée Territoriale de Corse. 
Mais plutôt que de revenir sur ce que fut la réalité historique des pieve, il nous semble plus pertinent pour la compréhension du fait insulaire de nous intéresser à la représentation collective qu'elles véhiculent dans l'imaginaire corse.

\subsubsection{Une partition Nord-Sud toujours prégnante}

Chaque région (de la Corse) a de fortes particularités. Que l'on pense au Cap Corse, totalement tourné vers le continent voisin, à la population composée d'agriculteurs/transporteurs. Mais que l'on pense aussi à la Balagne, à la Castagniccia, au Nebbio, au Cortenais, au Vicolais, au Sartenais, etc., toutes différentes. S'y surajoute un problème culturel et donc politique important, symbolisé par l'adoption dès le Moyen-Âge de la partition Nord-Sud (Deçà/Delà des Monts). À la fin du XVI ${ }^{e}$ siècle, le Deçà est deux fois plus peuplé que le Delà ; c'est un pays à dominante agraire, plus « civilisé " dans l'esprit d'un continental, où les Génois possèderont des amis sûrs et des alliés puissants. Le Delà est, au contraire, un espace montagnard rude, essentiellement pastoral, qui a longtemps figuré la résistance seigneuriale à l'influence génoise. Notons toutefois que les révolutions de Corse naitront au Nord et qu'elles se développeront de manière séparée, même au temps de Pascal Paoli. Cette partition gardera d'ailleurs son sens jusqu'à une période récente ${ }^{106}$.

Cette partition historique de l'île perdure dans la représentation collective des îliens et c'est un argument auquel ils ont recours pour évoquer le fait que la Corse n'est pas un tout uniforme mais que la frontière naturelle formée par ses chaines de montagnes a eu et a toujours des répercussions sur l'organisation sociale de l'île. Elle est d'autant plus présente dans la représentation que les insulaires ont de leur territoire de vie que l'enclavement s'est traduit par un faible développement des réseaux de transports qui, de manière récursive, a renforcé l'isolement des vallées et le sentiment de particularisme de chacune. Jean-François Battini ${ }^{107}$, constate :

La Corse, on dit parfois que c'est un archipel ; je crois que c'est là une des meilleures façons de la définir. [...] Chaque micro-région fonctionne un petit peu à sa manière et conçoit son développement et sa vie à partir d'elle-même. Et quand on franchit les frontières de la

110 Antoine-Marie Graziani, "Richesses et pesanteurs de l'Histoire 》, Confluences Méditerranée : Corse, les enjeux de l'après-Matignon, $\mathrm{n}^{\circ}$ 36, hiver 2000-2001, p. 34-35.

107 Alors directeur de cabinet de José Rossi, président de l'Assemblée territoriale de Corse (1999-2004) et membre du groupe Le Rassemblement. 
micro-région, on a l'impression qu'on passe vraiment ailleurs, et c'est comme ça que les gens le vivent, même si la télévision et ceci-cela font qu'il y a quand même une uniformisation. Mais, à mon sens, ces clivages sont réels, et ne serait-ce qu'entre Haute-Corse et Corse-duSud. Vizzavona, ce n'est pas qu'une barrière géographique ${ }^{108}$ dans la tête des gens. Pour les gens d'Ajaccio, forcément, Bastia, c'est le bout du monde, et vice versa, et pas que le bout du monde géographiquement. [...] On va dire qu'on ne les comprend pas, etc., qu'on n'a pas les mêmes objectifs.

Cette différence entre Ajaccio et Bastia est évoquée, de façon réciproque, par Jean-Guy Talamoni :

Évidemment entre un Bastiais et un Ajaccien, on dit que ce n'est pas la même mentalité, on dit que les Ajacciens sont plus, je dirais, traditionnellement, qu'ils sont plus démonstratifs, qu'ils ont plus le goût pour la vie publique, et pour se montrer, pour l'ostentation. C'est un petit peu ce qu'on dit et que les Bastiais sont plus travailleurs, plus industrieux, etc. Bon, les choses tendent à évoluer et c'est vrai qu'aujourd'hui les Bastiais se vivent un peu comme les Ajacciens et/ou l'inverse.

Cette partition de l'île a eu et a encore des conséquences sur l'organisation sociale et politique de la Corse. On peut voir dans le clanisme corse l'expression de cette séparation entre le Nord et le Sud de l'île.

Les réseaux de relation en Corse se conçoivent comme une forme de sociabilité particulière à la société insulaire. Cette sociabilité s'appuie sur les clans, c'est-à-dire les parentèles, les alliés d'une famille au sens large, à laquelle est associé un territoire, pris comme zone d'influence et d'exercice d'un pouvoir plus ou moins symbolique. L'instrument de cette organisation clanique est le clientélisme, c'est-à-dire l'échange de services entre membres du clan. Cependant il serait faux de penser que le clanisme est une spécificité corse, c'est un mode d'organisation sociopolitique répandu dans l'ensemble du bassin méditerranéen, que ce soit en Italie (Sardaigne, Sicile, etc.) ou au Maghreb ${ }^{109}$.

108 Le col de Vizzavona, sur la N193 qui relie Bastia à Ajaccio (ou Ajaccio à Bastia, selon les points de vue), est la frontière naturelle que l'on franchit et qui sert de démarcation entre les deux départements.

${ }^{109}$ Pour plus de développement sur ce point, voir Jean-Louis Briquet, La tradition en mouvement. Clientélisme et politique en Corse, Paris, Éditions Belin, Socio-Histoires, 1997, ainsi que Claude Olivesi, Le système politique corse dans sa nature méditerranéenne, thèse de $3^{\mathrm{c}}$ cycle en science politique à l'Université d'Aix Marseille III, 1982, sous la direction 
«Dénommé en corse partitu, le clan devint le pivot d'une organisation politique informelle et inavouée édifiée sur un rapport de dépendance entre, d'une part, les communautés villageoises et familiales, d'autre part, une personnalité dotée d'un pouvoir politique ${ }^{110}$. Ce mode de relation s'incarne dans le système politique, dans le clientélisme, défini : " comme un échange réciproque de services entre des individus de statuts sociaux inégaux (le "patron" et ses "clients"). Sous sa forme politique, il consiste en une relation fondée sur l'échange de biens matériels (emplois, subventions, aides diverses) contre un soutien électoral accordé par ses alliés à un notable ${ }^{111}$.

Le clientélisme illustre une vision du politique qui met en relation les liens de nature privée avec. un système politique moderne fondé sur la représentation et l'élection des représentants des communautés locales (communes, départements, cantons, régions, nation). En effet, en Corse, s'il peut y avoir concordance ou superposition des appartenances familiales et politiques, c'est que les cadres moraux qui les motivent sont de même ressort : fidélité au groupe et obligation morale de réciprocité. Les individus en Corse sont inscrits dans des réseaux relationnels et situationnels qui sont fortement imbriqués.

Les identités sociales et politiques des individus s'élaborent au sein de ces réseaux ; l'accès aux ressources gérées et distribuées par les élus en est fortement dépendant. Les réseaux se constituent à l'intérieur des communautés villageoises selon des logiques avant tout familiales. L'appartenance à un groupe familial reste le principal moyen d'identification d'un individu dans l'espace villageois ${ }^{112}$.

Cette appartenance perdure puisque de nombreux exilés continuent à voter dans leur village (ceci n'est pas pour faciliter le travail de lutte contre la fraude électorale). Jean-Louis Briquet rapporte un extrait

du professeur Bentz. Notons également que c'est un système que l'on retrouve généralement sur d'autres territoires isolés aux ressources limitées et avec des solidarités familiales fortes. C'est pourquoi on le rencontre en Albanie et, dans une certaine mesure, au Japon. C'est aussi un système qui peut s'exporter dans les phénomènes de migration.

110 Philippe Franchini, Les Corses, Paris, Le Cavalier Bleu éditions, Idées Reçues, 2001, p. 50.

111 Jean-Louis Briquet, La tradition en mouvement..., op. cit., p. 7.

112 Ibid., p. 63. 
d'entretien qu'il a eu avec une jeune femme qui, vivant et travaillant à Paris, avait été radiée en 1990 des listes électorales de son village où pourtant elle retourne très souvent :

Moi, voter à Paris, je m'en fous... Je ne sais même pas qui est le maire de mon arrondissement et je ne veux pas le savoir. Ici, je sais pour qui je vote, i'ai toute ma famille ; j'ai beaucoup d'amis... Quand je vote, je sais ce que ça veut dire, je vote pour quelqu'un que je connais depuis toujours, que je peux voir quand je veux... Ici, $j$ 'ai une maison, $j$ 'ai mes racines. Et je ne vois pas pourquoi Joxe m'interdirait de voter au village $^{113}$.

Les solidarités sont très importantes et il n'est pas rare qu'on trouve des gens votant pour telle personnalité politique en reconnaissance d'un service rendu à la famille des années plus tôt. "Ce qui compte, c'est paese e semiziu" ci deve rende unu semiziu, "il faut qu'on rende un service" ", nous a déclaré un élu évoquant la persistance du système clanique en Corse.

De plus, le rôle central de la famille permet de comprendre l'établissement de dynasties politiques qui assurent, pour le reste de la communauté, une visualisation et une identification nettes des réseaux sociaux et politiques ${ }^{115}$. Ces dynasties fonctionnent autour de « figures emblématiques, Emmanuel Arène au XIX ${ }^{c}$ siècle, François Pietri, Jacques Gavini entre les deux guerres, Paul Giacobbi ${ }^{116}$, Jean-Paul de Rocca-Serra, après la deuxième ${ }^{117}$. Il faut ici noter que :

le système est bipolaire, fondé sur l'antagonisme du partitu et du contrapartitu (parti minoritaire) : deux clans, le plus souvent l'un au nord

113 Ibid., p. 66. Cependant ce comportement ne nous semble pas spécifique aux Corses car nous pourrions le retrouver chez d'autres exilés régionaux ainsi que chez certains étudiants.

114 Littéralement «le pays, [le village] et le service », comme une sorte de devise.

115 Emmanuel Bernabeu-Casanova a mené une recherche très intéressante sur le comportement des élus d'origine corse en région d'Île-de-France qui reproduisent certaines conduites qui ont lieu sur l'île : «De l'île de Corse à l'île de France, les élus d'origine corse à Paris et dans les Hauts-de-Seine », Hérodote : revue de géographie et de géographie politique, Nationalismes régionaux en Europe, $\mathrm{n}^{\circ} 95,4^{\mathrm{c}}$ trimestre 1999, p. 114-144.

116 Après sa disparition, c'est son fils François qui reprend le flambeau.

117 Philippe Franchini, Les Corses, op. cit., p. 50-51. 
et l'autre au sud, s'opposent. [Pour ce qui est de l'inscription villageoise, notons que la famille Giacobbi vient de Venaco, à proximité de Corte et que la famille De Rocca-Serra vient de Porto-Vecchio.] Chacun est soutenu par une multitude de clans locaux d'importance variable, qui eux-mêmes s'emploient à reproduire en leur sein la transmission familiale des mandats électifs ${ }^{118}$.

Cela est particulièrement vérifié pour ce qui est des mandats municipaux qui très souvent se transmettent de père en fils (ou en fille), d'époux à épouse (c'est très souvent ce qui se passe en cas de décès).

Même si un renouvellement partiel du personnel politique insulaire avec l'émergence de nouvelles figures comme les nationalistes ou encore José Rossi a pu menacer à un moment le système clanique, force est de constater que celui-ci n'a pas disparu et qu'il reste ancré dans l'organisation sociopolitique corse ${ }^{119}$.

Pour Xavier Crettiez, «si l'État n'a pas créé le clan, il a considérablement aidé son développement $»^{120}$. Il apparaît que clans et État ont connu un développement parallèle, au fur et à mesure que s'affirmait la tutelle administrative de la France sur la Corse. Même si chacun possède sa propre logique de fonctionnement, ils participent à la construction d'un système politique corse spécifique, dont ils sont, tous les deux, des rouages nécessaires, inséparables et pourtant contradictoires, caractéristique qui fait donc de ce système un système complexe au sens morinien du terme. Aussi pouvons-nous voir que le système politique corse organisé autour de deux clans fortement intégrateurs relève de ce qu'Edgar Morin appelle les systèmes auto-éco-re-organisateurs.

Un système auto-organisateur est un système ouvert sur son environnement, dans lequel il puise énergie et information, et qui allie déterminisme interne et liberté propre. Pour Edgar Morin, «l'auto-

118 Jean-Louis Andreani, Pour comprendre la Corse, op. cit., p. 79.

119 On peut poser comme hypothèse que l'organisation bipolaire classique du clanisme autour du partitu et du contrapartitu, s'affrontant selon un rituel nommé la pulichitella (expression corse pour désigner la politique politicienne, c'est-à-dire des affrontements politiques stériles), a pu être remise en cause par le processus de Matignon en ce sens où on peut y voir une recomposition des alliances classiques, voire des retournements d'alliances : l'exemple le plus flagrant étant la position de Paul Giacobbi (qui a succédé à son père François) et qui a rejoint le camp des autonomistes alors que son père était farouchement pro-républicain.

120 Xavier Crettiez, La question corse, op. cit., p. 54. 
organisation est une notion clé qui implique l'auto-production, l'autoperpétuation, l'auto-reproduction, l'autorégénération à travers obstacles et aléas ${ }^{121}$. Or, ces éléments nous semblent présents dans la conception du clanisme telle que nous l'avons vue jusqu'à présent. Mais cette autonomie du système va de pair avec une forte dépendance à l'écosystème dans lequel le système étudié prend place. Ainsi pour le système clanique, son existence est fortement corrélée au pouvoir qu'il détient sur le système social insulaire grâce aux subsides qu'il reçoit du continent et qu'il redistribue à ses clientèles et ses parentèles. C'est pourquoi on ne peut non plus déconnecter la compréhension des problèmes corses du lien entre l'île et le continent. Vouloir comprendre les uns sans considérer l'autre serait un aveuglement pour l'entendement humain. Or,
l'idée d'auto-éco-organisation suppose l'idée de réorganisation permanente, $[\ldots]$ pour maintenir la constance ou l'homéostasie du système, en dépit et à travers le renouvellement constant de ses constituants (les molécules et les cellules d'un organisme se dégradent incessamment et sont reconstituées, les individus des sociétés meurent et sont renouvelés). C'est le paradoxe d'une organisation qui maintient ses structures, en dépit et à travers le renouvellement ininterrompu, le turnover de ses constituants ${ }^{122}$.

Cette partition a également des impacts au moment du choix de la situation géographique de certains équipements ou administrations. Quand la création de l'université de Corte fut acquise, la question se posa de savoir où la situer. Le gouvernement envisagea de l'installer à Ajaccio, mais finalement Corte, lieu historique de la première université, au temps de Pasquale Paoli, fut choisie, ce qui permit de mettre fin à une polémique dans le milieu régionaliste et autonomiste. De même, le fait que Ajaccio ait été choisie comme capitale territoriale a pu susciter quelques animosités, de peur que le sud de l'île ne soit favorisé par rapport au nord.

Nous avons eu l'occasion de voir au cours des paragraphes précédents que les acteurs évoquent une identité corse globale, mobilisable face à d'autres identités extérieures à la Corse et qui permettent de s'en différencier. Toutefois, cette identité globale,

\footnotetext{
121 Edgar Morin, Sociologie, Paris, Seuil, 1984, p. 102.

122 Ibid., p. 104.
} 
collective s'appuie sur des expériences diverses de la corsitude, laquelle s'appuie sur des identités vécues qui n'ont pas toutes la même réalité, la même substance. Il est vrai que vivre dans un village isolé de la montagne ou dans la plaine bastiaise ou encore à Ajaccio ne constitue pas la même expérience d'un vécu corse, au-delà des référentiels communs déjà évoqués (langue, culture, histoire).

Il semble donc vain de vouloir construire un idéal-type de l'identité corse tant elle revêt de formes : elle est vécue dans l'insularité mais elle est tout aussi forte dans sa forme diasporique ; elle se vit très bien dans le collectif mais fonctionne tout autant dans l'individualité et en dehors du rapport au groupe. C'est pourquoi on peut dire que l'identité corse est autre que la somme des référents dont on la gratifie. Elle n'est plus une identité singulière (et au singulier) mais des identités plurielles (et au pluriel). L'utilisation du pluriel est pertinente quand nous nous référons aux discours des élus locaux interrogés au cours de notre travail de terrain. Pour expliciter et justifier son action et ses positions, chacun va se référer à son village, à la communauté avec laquelle il a développé le sentiment de proximité le plus fort. Le singulier va référer à l'identité vécue dans une dimension globale. Identités plurielles et identité singulière ne peuvent pas être envisagées comme deux faits séparés, elles sont nécessairement liées. Cette dialogicité de l'identité illustre les deux faces du processus identitaire qui sont l'identification et l'individuation : c'est-à-dire que je considère les référentiels du groupe qui m'est proche (géographiquement, culturellement, familialement...) et que je les fais miens, mais, ce faisant, i’y imprime également ma personnalité, puisqu'ils passent par le prisme de mon regard.

Ceci rend donc légitime notre interrogation quant à l'importance de la relation au territoire dans l'identité globale corse. Relation qui d'ailleurs amène à ne pas prendre comme référent unique le territoire propre (l'île) mais aussi d'autres territoires, plus ou moins proches, plus ou moins lointains, tant sur le plan géographique que culturel, etc. Le processus identitaire, c'est-à-dire la mise en place et la construction de l'identité, sa définition, se crée dans une relation avec des territoires qui sont eux-mêmes en contact, en interactions et en interrelations, comme nous l'avons abordé concernant les liens qui unissent la Corse à la Méditerranée, à la France, à l'Italie mais à l'Europe aussi. 


\section{Conclusion}

Au terme de cet article, et à partir des arguments que nous avons mobilisés à partir de l'exemple corse, nous considérons qu'il nous faut revenir sur la notion même d'identité pour la considérer comme une notion complexe, au sens morinien. Elle est le lieu d'une auto-éco-redéfinition, c'est-à-dire qu'elle repose sur trois dimensions essentielles, qui interagissent entre elles.

Toute identité collective est un soi-disant. Elle comporte une autodéfinition : je me pense comme élément d'un groupe qui se pense luimême comme une entité (quelle qu'elle soit : entreprise, parti politique, groupe d'amis, famille au sens large...) avec des caractéristiques générales que chaque élément va s'approprier, jusqu'à former une communauté (d'action, d'intérêt, de vie, de destin, d'affectivité....). L'identité est donc un vécu, une expérience : l'individu se ressent comme membre de cette communauté, il appartient à cette communauté.

L'identité est aussi, bien entendu, un rapport aux autres, l'individu ne peut appartenir à une communauté si les autres membres ne le reconnaissent pas comme un des leurs.

L'identité donne un sens à l'existence. Dès lors, la question de l'identité revêt une dimension sociale, du fait que l'existence ne saurait se réduire à sa dimension singulière [...]. L'identité dont nous sommes porteurs et qui fait de nous des sujets se fonde et se construit dans le rapport à l'autre qui fait de nous des sujets en nous nommant et en nous reconnaissant l'existence ${ }^{123}$.

En ce sens l'identité repose également sur une éco-définition, c'est-à-dire quelque chose qui se définit en fonction d'un environnement au sens large : humain, géographique, économique, historique... Dans sa dimension collective, elle est la construction d'un sentiment d'appartenance à une communauté, parfois attachée à un lieu, c'est-à-dire un environnement physique, matérialisé, particulier : un territoire. Et cette relation au lieu peut être vécue différemment selon les spécificités du territoire concerné.

La dimension territoriale est, avec le sentiment d'appartenance communautaire, l'un des aspects que nous avons privilégiés dans notre approche. Pour notre part, nous nous situons dans une approche du territoire telle que développée par Yves Barel et qui ne nous semble

123 Bernard Lamizet, Politique et identité, Lyon, Presses universitaires de Lyon, 2002, p. 5. 
restrictive d'aucun sens ni d'aucune expérience. Pour lui, le territoire est « un milieu de vie, de pensée et d'action dans lequel et grâce auquel un individu ou un groupe se reconnait, dote ce qui l'entoure de sens et se dote lui-même de sens, met en route un processus identificatoire et identitaire $^{124} \gg$. L'identité se définit en ce sens dans un rapport à un territoire (et à son histoire), par rapport aux autres et par rapport au regard que les autres ont sur soi (ou du moins ce qu'on en ressent ou ce qu'ils en disent). Le regard des autres est important car c'est face à lui qu'on va éprouver la force de l'identité que l'on s'est construit, tant sur le plan individuel que collectif.

D'où l'idée que l'identité est également un processus de redéfinition permanente, c'est-à-dire, qu'elle n'est pas figée, statique. Son dynamisme fait qu'elle est amenée à évoluer, à se modifier en intégrant les changements sociétaux, globaux ou particuliers.

Cette identité est toujours en transformation puisque les contextes de
référence de cette identité : contexte biologique, psychologique, temporel,
matériel, économique, relationnel, normatif, culturel, politique... qui
fournissent les significations, sont chacun en évolution. Elle est, à un
moment donné, la résultante d'un ensemble d'autoprocessus (génétiques,
biologiques, affectifs, cognitifs...) et de processus (relationnels et
communicationnels, historiques, culturels...) formant entre eux un
système de causalités circulaires. Elle est donc toujours un construit $[. . .]^{125}$.

C'est donc dire qu'en se confrontant à l'Autre, au regard qu'il porte sur nous, nous sommes amenés à nous positionner par rapport à ce regard et à nous redéfinir continuellement. Nous pouvons alors lire l'identité comme étant à la fois ce processus et le résultat de ce processus de construction de soi.

C'est pourquoi nous considérons que l'identité est une auto-éco-redéfinition d'un individu ou d'un groupe, d'une communauté par rapport à d'autres et par rapport à un environnement. Nous pouvons dès lors la voir comme une propriété émergente d'un système social. Les "propriétés émergentes » d'un système désignent « les qualités ou propriétés d'un système qui présentent un caractère de nouveauté par

124 Yves Barel, “Territoires et corporatismes », Économie et Humanisme, n 314, 1990, cité par Philippe Tizon, "Qu'est-ce que le territoire? ", dans Guy Di Meo (dir.), Les territoires du quotidien, op.cit, p. 21.

125 Alex Mucchielli, L'identité, Paris, PUF, Que sais-je ?, 1999, p. 10. 
rapport aux qualités ou propriétés des composants considérés isolément $»^{126}$. Elles peuvent faire apparaitre d'autres propriétés : l'identité est alors elle-même créatrice d'identité.

\section{Références}

Albertini, Jean-Vitus et Paul-François Torre, Jospin : le pari corse. Histoire du processus de Matignon, Ajaccio, Albiana Éditions, 2002.

Andreani, Jean-Louis, Pour comprendre la Corse, Paris Folio-Actuel, Le Monde, 1999.

Arrighi, Paul et Francis Pomponi, Histoire de la Corse, Paris, PUF, Que sais-je ?, 1993.

Barel, Yves, «Le social et ses territoires », dans François Auriac et Roger Brunet (dir.), Espaces, jeux et enjeux, Paris, Fayard, 1986, cité par Philippe Tizon, «Qu'est-ce que le territoire ?", dans Guy Di Meo (dir.), Les territoires du quotidien, Paris, L'Harmattan, Géographie sociale, 1996.

Barel, Yves, "Territoires et corporatismes », Économie et Humanisme, $n^{\circ} 314,1990$, cité par Philippe Tizon, "Qu'est-ce que le territoire? ", dans Guy Di Meo (dir.), Les territoires du quotidien, Paris, L'Harmattan, Géographie sociale, 1996.

Bernabeu-Casanova, Emmanuel, Le nationalisme corse : genèse, succès et échec, Paris, L'Harmattan, 1997.

Bernabeu-Casanova, Emmanuel, «De l'île de Corse à l'île de France, les élus d'origine corse à Paris et dans les Hauts-de-Seine », Hérodote : revue de géographie et de géographie politique, $\mathrm{n}^{\circ}, 95,4^{\mathrm{e}}$ trimestre 1999 , p. 114-144.

Bernabeu-Casanova, Emmanuel et Dominique Lanzalavi, Corse, les voies de l'avenir, Paris, L'Harmattan, 2003.

Briquet, Jean-Louis, La tradition en mouvement. Clientélisme et politique en Corse, Paris, Éditions Belin, Socio-Histoires, 1997.

Bromberger, Christian et Mireille Meyer, "Cultures Régionales en débat », Etbnologie francaise, "Cultures régionales. Singularités et revendications ", vol. XXXIII, n 3 , 2003, p. 357-361.

Caratini, Roger, Histoire du peuple corse, Paris, Criterion, Histoire et histoires, 1995.

Caratini, Roger, La Corse, un peuple, une bistoire, Paris, Éditions de l'Archipel, 2003 (édition revue et augmentée de l'Histoire du peuple corse, Paris, Éditions Criterion, 1995).

Carrington, Dorothy, La Corse, Paris, Arthaud, 1999.

Casta, Ange (dir.), La parabole corse, rencontres avec l'identité, Ajaccio, Éditions Albiana, 1995.

Castelain, Jean-Pierre, «Insularités », Etbnologie Française, vol. XXXIV, n 1, 2004, p. 99 100.

126 Edgar Morin, La Méthode, tome I, La Nature de la Nature, Paris, Seuil, 1977, p. 106. 
Centre Européen des questions de minorités, Autonomies insulaires, Ajaccio, Éditions Albiana, 1999.

Chemins d'étoiles, «Îles funestes, îles bienheureuses », Paris, Éditions Transboréal, n 12 , août 2004.

Colombani, Jean-Marie, Les infortunes de la république, Paris, Grasset, 2000.

Confluences Méditerranée, "Corse : les enjeux de l'après-Matignon », Paris, L'Harmattan, $\mathrm{n}^{\circ}$ 36, hiver 2000-2001.

Crettiez, Xavier, La question corse, Bruxelles, Éditions Complexe, 1999.

De Casablanca, François, « Méditerraniser l'Europe », Corsica Magaz̧ine, août 2001, p. 66.

Desanti, Jean-Toussaint, «Les lieux sont tombés sur la tête », dans Ange Casta (dir.), La parabole corse, rencontres avec l'identité, Ajaccio, Éditions Albiana, 1995, p. 10-14.

Desanti, Jean-Toussaint, "Effacer la mer. Une réflexion sur l'identité corse », Esprit, Dossier la Fièvre identitaire, janvier 1997, p. 148-155.

Di Meo, Guy (dir.), Les territoires du quotidien, Paris, L'Harmattan, Géographie sociale, 1996.

Doumenge, François, «Les îles et les micro-états insulaires », Hérodote : revue de géographie et de géographie politique, $\mathrm{n}^{\circ \mathrm{s}} 37-38,2^{\mathrm{e}}$ et $3^{\mathrm{e}}$ trimestres $1985, \mathrm{p} .297-327$.

Dressler, Wanda, La Corse en question(s), Ajaccio, Albiana, 2004.

Fabre, Thierry (dir.), La Méditerranée créatrice, La Tour d'Aigues, Éditions de l'Aube, 1994.

Franchini, Philippe, Les Corses, Paris, Le Cavalier Bleu éditions, Idées Reçues, 2001.

Giblin, Béatrice, «Les nationalismes régionaux en Europe », Hérodote : revne de géographie et de géographie politique, $\mathrm{n}^{\circ} 95,4^{\mathrm{e}}$ trimestre 1999 , p. 3-20.

Graziani, Antoine-Marie, «Comment pcut-on être Corse? " Commentaire, Éditions Julliard, vol. $20, \mathrm{n}^{\circ} 80$, hiver 1998 , p. 929-939.

Graziani, Antoine-Marie, "Richesses et pesanteurs de l'Histoire ", Confluences Méditerranée : Corse, les enjeux de l'après-Matignon, $n^{\circ}$ 36, hiver 2000-2001, p. 3144.

Guillaud, Dominique, Christian Huetz de Lemps et Olivier Sevin (dir.), Îles rêvées. Territoires et identités en crise dans le Pacifique insulaire, Paris, Presses de l'Université de Paris-Sorbonne, 2003.

Huetz de Lemps, Christian, "Des hommes et des îles : l'originalité du Pacifique ", Chemins d'étoiles, Îles funestes, îles bienheureuses, Paris, Éditions Transboréal, $n^{\circ} 12$, août 2004, p. 78-91.

La Méditerranée, modernité plurielle, Paris, Éditions Publisud, 2000.

Lamizet, Bernard, Politique et identité, Lyon, Presses universitaires de Lyon, 2002.

Lampedusa, Guiseppe Tomasi di, Le Guépard, Paris, Seuil, 1959.

Lapidoth, Ruth, «Les caractéristiques de l'autonomic », dans Centre Européen des questions de minorités, Autonomies insulaires, Ajaccio, Éditions Albiana, 1999, p. $27-$ 33.

Lefevre, Marianne, Géopolitique de la Corse, le modèle républicain en questions, Paris, L'Harmattan, Histoire en perspective, 2000.

Letoublon, Françoise (dir.), Impressions d’lles, Toulouse, Presses Universitaires du Mirail, 1996. 
Letoublon, Françoise, Paola Ceccarelli et Jean Sgard, "Qu'est-ce qu'une île ? », dans Françoise Letoublon (dir.), Impressions d'îles, Toulouse, Presses Universitaires du Mirail, 1996, p. 9-27.

Loughlin, John, "Introduction », dans Centre Européen des questions de minorités, Autonomies insulaires, Ajaccio, Éditions Albiana, 1999, p. 9-17.

Lugan, Jean-Claude, La systémique sociale, Paris, PUF, Que sais-je ?, 1993.

Maccioni, Pascal, "La Corse : la plus grecque des terres françaises », Confluences Méditerranée, $\mathrm{n}^{\circ} 4$, Automne 1992, http://www.ifrance.com/Confluences/textes/ 4 maccioni.htm, consulté en ligne le 25 juin 2003.

Maroselli-Matteoli, Marie-Catherine, La reconquête de la dignité, Ajaccio, La Marge Éditions, 1990.

Martinetti, Joseph, Insularité et marginalité en Méditerranée occidentale : l'exemple de la Corse, Ajaccio, Le Signet, 1991.

Meistersheim, Anne (dir.), L'île laboratoire, Colloque de l'Université de Corse, 19-21 juin 1997, Ajaccio, Éditions Alain Piazzola.

Meistersheim, Anne, Figures de l'île, Ajaccio, DCL Éditions, 2001.

Molinelli-Cancellieri, Lucia, Boues Rouges, la Corse dit non, Paris, L' Harmattan, 1995.

Morin, Edgar, La Méthode, 6 tomes, 1977-2004, Paris, Seuil.

Morin, Edgar, La Méthode, tome I, La Nature de la Nature, Paris, Seuil, 1977.

Morin, Edgar, Sociologie, Paris, Seuil, 1984.

Morin, Edgar, La Méthode, tome 5, L'bumanité de l'bumanité. L'identité humaine, Paris, Seuil, 2001.

Mucchielli, Alex, L'identité, Paris, PUF, Que sais-je ?, 1999.

Olivesi, Claude, Le système politique corse dans sa nature méditerranéenne, Thèse de $3^{\mathrm{e}}$ cycle en science politique à l'Université d'Aix Marseille III, 1982, sous la direction du professeur Bentz.

Olivesi, Claude, «La Corse contestataire, l'État et l'Europe », dans Fred Reno (dir.), Identité et politique. De la Caraïbe et de l'Europe multiculturelles, Paris, Economica, Caraibe - Amérique Latine, 1995, p. 155-201.

Orsoni, Claude, "Clanisme et racisme. Hypothèses sur les relations intercommunautaires en Corse ", Peuples Méditerranéens, $n^{\circ}$ 51, Le néo-racisme en Corse, avril-juin 1990, p. 191-201.

Poggioli, Jackie, «De la féminité de l'identité corse », dans Ange Casta (dir.), Laparabole corse, Ajaccio, Éditions Albiana, 1995, p. 100-103.

Ravis-Giordani, Georges, "L'impossible Mezzogiorno ", Méditerranéennes, Éditions Albiana, Association Méditerranéennes, $n^{\circ} 12$, été 2001, p. 28-39.

Ravis-Giordani, Georges, «La Corse : culture régionale ? Culture régionalisée ?», Ethnologie Française, "Cultures régionales. Singularités et revendications », vol. XXXIII, n 3, 2003, p. 451-458.

Renucci, Janine, La Corse, Paris, PUF, Que sais-je?, 2001.

Rombaldi, Michel, "La coopération interrégionale en Méditerranée », dans Anne Meistersheim (dir.), Lîle laboratoire, Ajaccio, Éditions Alain Piazzola, 1999, p. 109. 122.

Roux, Michel, Inventer un nouvel art d'babiter : le ré-enchantement de l'espace, Paris, L'Harmattan, 2002. 
Roux, Michel, Le Ré-enchantement du territoire, publié en mars 2001 sur le site http://www.mcxapc.org/ consulté en mars 2003.

Salini, Dominique, "L'île entre laboratoire et lab-oratoire ou de la complexité de la mesure », dans Anne Meistersheim (dir.), L'île laboratoire, Colloque de l'Université de Corse, 19-21 juin 1997, Ajaccio, Éditions Alain Piazzola.

Sanguin, André-Louis (dir.), Vivre dans une ille. Géopolitique des insularités, Paris, L'Harmattan, Géographie et cultures, 1997.

Silvani, Paul, Corse des années ardentes (1939-1976), Paris, Éditions Albatros, 1976.

Siméoni, Edmond, Un combat pour la Corse. Entretiens avec Pierre Dottelonde, Paris, Le Cherche midi, Documents, 2003.

Sylvestre, Françoise, "L'âme vagabonde, d'une île à l'autre ", Chemins d'étoiles, Paris, Éditions Transboréal, $n^{\circ} 12$ «Îles funestes, îles bienheureuses », août 2004, p. 8-14.

Talamoni, Jean-Guy, Ce que nous sommes. Cio che no simu, Péface d'Albert Memmi, Ajaccio, Éitions DCL, Éditions Ramsay, 2001.

Tillard, Delphine, "La Bataille de Ponte-Novo. Liturgie d'une défaite ?, Socioanthropologie, $\mathrm{n}^{\circ} 9$, Commémorer. Consulté sur le site : http://revel.unice.fr/ anthropo/document.html?id $=10$

Verdoni, Dominique, "L'insularité : approche herméneutique de la variation ", dans Anne Meistersheim (dir.), L'île laboratoire, Colloque de l'Université de Corse, 19-21 juin 1997, Ajaccio, Éditions Alain Piazzola, p. 436-442

Verge-Franceschi, Michel, Histoire de la Corse, le pays de la grandeur, Préface d'Emmanuel Leroy-Ladurie, Paris, Éditions du Félin, 1996.

Villa, Paul-Michel, La maison des Viale, Ajaccio, Édition Alain Piazzola, 1994.

www.livre-insulaire. fr $\backslash$ index.html. 